\title{
GROUPS OF AUTOMORPHISMS OF BOREL SPACES
}

\author{
BY \\ V. S. VARADARAJAN(1)
}

1. Introduction. The main object of the present paper is to describe a generalization of some aspects of the classical von Neumann-Krylov-Bogoljubov theory of the decomposition of a suitably restricted Borel space into its so-called ergodic parts relative to a flow $[1 ; 13 ; 18 ; 19]$. Our main results deal with locally compact groups of transformations acting measurably on sufficiently smooth Borel spaces, and lead to a fairly detailed picture of the action of such groups of automorphisms.

The organization of the present paper is as follows. In $\$ 2$ we summarize the basic material concerning Borel spaces which is pertinent to our subsequent discussions. In $\$ 3$ we introduce the notion of a Borel $G$-space and examine the intimate relation between Borel $G$-spaces and topological $G$-spaces. We obtain, among other things, generalizations of well-known results of Mackey [17] and Ambrose-Kakutani [2]. In $\$ 4$ we take up the study of a standard Borel $G$-space. We show that any standard Borel $G$-space can be decomposed, in an essentially unique fashion, into disjoint invariant Borel sets on each of which the group acts uniquely ergodically.

This decomposition leads to canonical forms for standard Borel $G$-spaces and to representations of invariant measures as integrals of ergodic measures. In $\$ 5$ we study the convex set of all invariant measures on an arbitrary Borel $G$-space. We prove that every invariant measure is uniquely determined by its values on the $\sigma$-algebra of invariant sets and that the set of invariant measures is isomorphic to the set of all measures on some, possibly abstract, $\sigma$-algebra. In $\S 6$ we give a number of examples and make a few remarks supplementing the preceding discussions.

Some of the results of this paper are contained in a research announcement of the Bulletin of the American Mathematical Society. After the announcement was submitted, the present writer came across a preprint of Dr. R. H. Farrell of Cornell University in which results close to some of the results of the present work have been obtained. This writer would like to express his warm thanks to Dr. Farrell for allowing a reference to his paper to be included in this work; Dr. Farrell's work will appear in the Illinois Journal of Mathematics. Thanks

Received by the editors June 4, 1962 and, in revised form, October 11, 1962.

(1) This work was done while the author was a temporary member of the Courant Institute of Mathematical Sciences during 1961-1962 under the parial support of the National Science Foundation. 
are also expressed to Dr. R. Sacksteder and Dr. H. Teicher of the Courant Institute of Mathematical Sciences, and to Dr. Ranga Rao of the University of Illinois, for their interest in the present work.

2. Borel spaces. In this section we shall summarize briefly the basic concepts concerning Borel spaces and prove certain auxiliary lemmas. Our terminology closely parallels that of Mackey ([17]; cf. also [4; 14]).

A Borel space is a pair $(X, \mathscr{B})$ where $X$ is an abstract set and $\mathscr{B}$ a $\sigma$-algebra of subsets of $X ; \mathscr{B}$ is called the Borel structure of the Borel space. When no confusion can arise we refer to $X$ itself as the Borel space. A Borel space $(Y, \mathscr{C})$ is a Borel subspace of the Borel space $(X, \mathscr{B})$ if $Y \subseteq X$ and $\mathscr{C}$ consists precisely of the sets of the form $A \cap Y$ with $A \in \mathscr{C}$; we then refer to it as the Borel subspace defined by $Y$. If $\left(X_{1}, \mathscr{B}_{1}\right)$ and $\left(X_{2}, \mathscr{B}_{2}\right)$ are Borel spaces, a map $f$ of $X_{1}$ into $X_{2}$ is called measurable if $f^{-1}(A) \in \mathscr{B}_{1}$ for all $A \in \mathscr{B}_{2}$. Two Borel spaces $\left(X_{1}, \mathscr{B}_{1}\right)$ and $\left(X_{2}, \mathscr{B}_{2}\right)$ are called isomorphic if there exists a one-to-one map $f$ of $X_{1}$ onto $X_{2}$ such that $f$ and $f^{-1}$ are both measurable; any such $f$ is called an isomorphism. An automorphism of a Borel space $(X, \mathscr{B})$ is a one-to-one map of $X$ onto itself such that $f^{-1}(A) \in \mathscr{B}$ if and only if $A \in \mathscr{B}$. If $X$ is a topological space we define the Borel space associated with $X$ to be the pair $(X, \mathscr{B})$ where $\mathscr{B}$ is the smallest $\sigma$-algebra of subsets of $X$ with respect to which all real-valued continuous functions are measurable. $\mathscr{B}$ is then the $\sigma$-algebra generated by sets of the form $f^{-1}(C)$ where $C$ is any closed subset of the real line and $f$ any continuous function on $X$. When $X$ is a metric space, $\mathscr{B}$ coincides with the $\sigma$-algebra generated by the class of all open sets in $X$; in this case we shall refer to sets of $\mathscr{B}$ as Borel sets.

A Borel space $(X, \mathscr{B})$ is countably generated if there exists a denumerable class $\mathscr{U} \subseteq \mathscr{B}$ such that $\mathscr{B}$ is the $\sigma$-algebra generated by $\mathscr{U}$; it is called separable if it is countably generated and if $\{x\} \in \mathscr{B}$ for each $x \in X .(X, \mathscr{B})$ is called standard if it is isomorphic to the Borel subspace of a complete separable metric space defined by a Borel set; it is called analytic if it is isomorphic to the Borel subspace defined by an analytic subset of a complete separable metric space. A separable Borel space $\left(X^{\prime}, \mathscr{B}^{\prime}\right)$ is analytic if and only if there exists a standard Borel space $(X, \mathscr{B})$ and a measurable map $f$ such that $f$ maps $X$ onto $X^{\prime}$.

It is a consequence of some deep theorems in the theory of Borel sets in metric spaces that any two standard Borel spaces are isomorphic if (and only if) the underlying spaces have the same cardinality; moreover the only uncountable cardinal that can arise is that of the continuum. In particular every standard Borel space whose underlying set is uncountable is isomorphic to the Borel space associated with the unit interval.

By a measure $\mu$ on a Borel space $(X, \mathscr{B})$ we understand a non-negative countably additive set function defined on $\mathscr{B}$ with $\mu(X)=1 . \mu$ is called standard if there exists an $N \in \mathscr{B}$ such that (i) $\mu(N)=0$, (ii) the Borel subspace defined by $X-N$ is standard. A Borel space is said to be metrically standard if every measure on it is 
standard. Any analytic Borel space is metrically standard. If $\mathscr{M}$ is the set of all measures on a Borel space $(X, \mathscr{B})$ and $\mathscr{M}^{\prime}$ any subset of $\mathscr{M}$; we denote by $\mathscr{A}\left(\mathscr{M}^{\prime}, \mathscr{B}\right)$ the smallest $\sigma$-algebralof subsets of $\mathscr{M}^{\prime}$ that makes all the maps $\mu \rightarrow \mu(A)$ $(A \in \mathscr{B})$ measurable. We call $\mathscr{A}\left(\mathscr{M}^{\prime}, \mathscr{B}\right)$ the customary Borel structure for $\mathscr{M}^{\prime}$. We shall usually write $\mathscr{A}$ for $\mathscr{A}\left(\mathscr{M}^{\prime}, \mathscr{B}\right)$ whenever the context makes it clear what $\mathscr{M}^{\prime}$ and $\mathscr{B}$ are; often we omit even mentioning $\mathscr{A}$. If $\mathscr{M}_{2} \subseteq \mathscr{M}_{1} \subseteq \mathscr{M}$ the Borel space $\mathscr{M}_{2}$ is the Borel subspace of $\mathscr{M}_{1}$ defined by $\mathscr{M}_{2}$.

When $X$ is a completely regular Hausdorff space, the space $\mathscr{V}$ of all finite countably additive set functions on the associated Borel space becomes a locally convex Hausdorff topological vector space when it is equipped with its weak*topology, i.e., the smallest topology on $\mathscr{V}$ which makes the maps $\tau_{g}: \phi \rightarrow \int_{X} g d \phi$ continuous $(\phi \in \mathscr{V})$ for all bounded continuous $g$ on $X$. Topological statements concerning $\mathscr{V}$ or its subsets are always to be understood with reference to this topology. When $X$ is a separable metric space and $\mathscr{M}$ is the set of all measures on the associated Borel space, $\mathscr{M}$ is a separable metric space. If $X$ is a complete separable metric space $\mathscr{M}$ can be given a complete metrization. $\mathscr{M}$ is a compact metric space if and only if $X$ is so $[20 ; 21 ; 22]$.

Finally we make a few remarks on countably generated Borel spaces. Such Borel spaces need not be separable. However, if $(X, \mathscr{B})$ is countably generated, then each $x \in X$ lies in a unique atom $A_{x}$ of $\mathscr{B}$, i.e., a non-null set in $\mathscr{B}$ such that if $A \in \mathscr{B}$ and $A \subseteq A_{x}$ then either $A=\emptyset$ or $A=A_{x}$. In this case, if $X^{0}$ is the set of all atoms, $\pi$ is the map $x \rightarrow A_{x}$, and $\mathscr{B}^{0}$ is the class of all sets $A^{0} \subseteq 1 X^{0}$ such that $\pi^{-1}\left(A^{0}\right) \in \mathscr{B}$, then $\left(X^{0}, \mathscr{B}^{0}\right)$ is a separable Borel space, called the canonical separable Borel space associated with $(X, \mathscr{B})$. If $\alpha$ is any automorphism of $(X, \mathscr{B})$ there is a unique automorphism $\alpha^{0}$ of $\left(X^{0}, \mathscr{B}^{0}\right)$ such that $\pi \circ \alpha=\alpha^{0} \circ \pi$. Moreover, the correspondence $\alpha \rightarrow \alpha^{0}$ is a homomorphism, i.e., $(\alpha \circ \beta)^{0}=\alpha^{0} \circ \beta$ and $\left(\alpha^{-1}\right)^{0}=\left(\alpha^{0}\right)^{-1}$.

Lemma 2.1. Let $(X, \mathscr{B})$ be a separable Borel space. Then there exists a standard Borel space $\left(X^{\prime}, \mathscr{B}^{\prime}\right)$ such that $X \subseteq X^{\prime}$ and $\mathscr{B}=\mathscr{B}^{\prime} \cap X$, i.e., $\mathscr{B}=\left\{A^{\prime} \cap X: A^{\prime} \in \mathscr{B}^{\prime}\right\}$. In particular, there is a separable metric topology for $X$ under which $\mathscr{B}$ is the class of all Borel subsets of $X$.

Proof. This is just Theorem 2.1 of [17].

LeMma 2.2. Let $(X, \mathscr{B})$ and $(Y, \mathscr{C})$ be analytic Borel spaces and let $\pi$ be a measurable map of $X$ onto $Y$. If $v$ is any measure on $\mathscr{C}$ there exists a measure $\mu$ on $\mathscr{B}$ such that $v(A)=\mu\left(\pi^{-1}(A)\right)$ for all $A \in \mathscr{C}$.

Proof. Since $X$ itself is the image under a measurable map of a standard space, there is no loss of generality in assuming that $X$ is standard. Suppose $X$ is standard. By a lemma of von Neumann [17, Theorem 6.3] there exists a set $N \in \mathscr{C}$ and a set $E \in \mathscr{B}$ such that (i) $v(N)=0$ (ii) $\pi$ is one-to-one on $E$ and $\pi[E]=Y-N$. Since the Borel subspace defined by $E$ is standard and $\pi$ is one-to-one, it follows that 
$\pi$ is an isomorphism between $E$ and $Y-N$. It now suffices to define the measure $\mu$ on $\mathscr{B}$ by setting $\mu(A)=v(\pi[A \cap E])$ for all $A \in \mathscr{B}$.

COROllary. Let $(X, \mathscr{B})$ be an analytic Borel space and let $\mathscr{B}^{\prime} \subseteq \mathscr{B}$ be a countably generated $\sigma$-algebra. If $\mu^{\prime}$ is any measure on $\mathscr{B}^{\prime}$, there exists at least one measure $\mu$ on $\mathscr{B}$ whose restriction to $\mathscr{B}^{\prime}$ coincides with $\mu^{\prime}$.

Proof. It is well known that there exists a real-valued function $f$ on $X$ such that $\mathscr{B}{ }^{\prime}=\left\{f^{-1}(E): E\right.$ a Borel set of the line $\}$. Let $Y=f[X]$. Then $Y$ is an analytic subset of the line. Let $(Y, \mathscr{C})$ be the Borel subspace of the line defined by $Y$ and for any $C \in \mathscr{C}$ write $v(C)=\mu^{\prime}\left(f^{-1}(C)\right)$. The corollary follows from the lemma at once.

Let now $X$ be a separable metric space and $\mathscr{B}$ the class of Borel subsets of $X$. The space $\mathscr{M}$ of all measures on $\mathscr{B}$ is a Borel space when equipped with what we have called its customary Borel structure $\mathscr{A}$. On the other hand, $\mathscr{M}$ is a separable metric space in its weak*-topology and hence we may consider the $\sigma$-algebra $\mathscr{A}^{\prime}$ of Borel sets of the separable metric space $\mathscr{M}$.

LEMMA 2.3. $\mathscr{A}=\mathscr{A}^{\prime}$.

Proof. We first show that $\mathscr{A} \subseteq \mathscr{A}^{\prime}$. If $\mathscr{L}$ is the class of all bounded measurable functions $g$ on $X$ for which the maps $\tau_{g}: \mu \rightarrow \int_{X} g d \mu$ are all $\mathscr{A}^{\prime}$-measurable, it is clear that $\mathscr{L}$ is closed under formation of linear combinations and under bounded pointwise convergence. Moreover, for any bounded continuous $g$, $\tau_{g}$ is continuous on $\mathscr{M}$ and hence $\mathscr{A}^{\prime}$-measurable. $\mathscr{L}$ thus contains all bounded measurable functions, in particular the characteristic functions of sets in $\mathscr{B}$. By the definition of $\mathscr{A}$ we now infer that $\mathscr{A} \subseteq \mathscr{A}^{\prime}$.

To show that $\mathscr{A}^{\prime} \subseteq \mathscr{A}$ it is enough to show that any open set in $\mathscr{M}$ belongs to $\mathscr{A}$. Since any bounded measurable function is the uniform limit of a sequence of linear combinations of characteristic functions, it follows that for each bounded measurable $g$, the map $\mu \rightarrow \int_{X} g d \mu$ is $\mathscr{A}$-measurable. Consequently, for any $\varepsilon>0$ and bounded continuous functions $g_{1}, g_{2}, \cdots, g_{k}$ on $X$, the set

$$
N\left(\mu_{0}: g_{1}, \cdots, g_{k} ; \varepsilon\right)=\left\{\mu: \max _{1 \leqq i \leqq k}\left|\int_{X} g_{i} d \mu-\int_{X} g_{i} d \mu_{0}\right|<\varepsilon\right\}
$$

lies in $\mathscr{A}$. The totality of all such sets is a base for the topology of $\mathscr{M}$, and since $\mathscr{M}$ is a separable metric space, each open set in $\mathscr{M}$ is a countable union of sets of the form (*), and is hence a member of $\mathscr{A}$. This iproves that $\mathscr{A}^{\prime} \subseteq \mathscr{A}$ and completes the proof of the lemma.

THEOREM 2.1. Let $(X, \mathscr{B})$ be a countably generated Borel space and $\mathscr{M}$ the space of all measures on $\mathscr{B}$. $\mathscr{M}$ is then a separable Borel space. If $X$ is standard, so is $\mathscr{M}$; if $X$ is analytic, so is $\mathscr{M}$.

Proof. Since the Borel structure of $\mathscr{M}$ depends only on the $\sigma$-algebra $\mathscr{B}$ and 
since the $\sigma$-algebra $\mathscr{B}^{0}$ is $\sigma$-isomorphic to $\mathscr{B}$ we may and do replace $(X, \mathscr{B})$ by the associated separable Borel space $\left(X, \mathscr{B}^{0}\right)$. In other words we shall assume that $(X, \mathscr{B})$ is separable. There is then some separable metric topology for $X$ under which $\mathscr{B}$ becomes the class of Borel subsets of $X$. The space $\mathscr{M}$ will then be a separable metric space and hence by Lemma $2.3(\mathscr{M}, \mathscr{A})$ is a separable Borel space, $\mathscr{A}$ being the customary Borel structure on $\mathscr{M}$.

Suppose now $(X, \mathscr{B})$ is standard. We may then assume that $X$ is a complete separable metric space and $\mathscr{B}$ the class of Borel subsets of $X$. $\mathscr{M}$ will then be a complete separable metric space in its weak*-topology and hence we infer from Lemma 2.3 that $\mathscr{M}$ is a standard Borel space.

Suppose finally that $X$ is analytic. Then there exists a standard Borel space $\left(X^{\prime}, \mathscr{B}^{\prime}\right)$ and a measurable map $\pi: X^{\prime} \rightarrow X$ such that $\pi\left[X^{\prime}\right]=X$. Let $\mathscr{M}^{\prime}$ be the space of all measures on $\mathscr{B}^{\prime}$ and for any $\mu^{\prime} \in \mathscr{M}^{\prime}$ let $\mu_{\pi}^{\prime} \in \mathscr{M}$ be defined by setting $\mu_{\pi}^{\prime}(A)=\mu^{\prime}\left(\pi^{-1}(A)\right)$ for all $A \in \mathscr{B} . \mu^{\prime} \rightarrow \mu_{\pi}^{\prime}$ is a measurable map of $\mathscr{M}^{\prime}$ into $\mathscr{M}$ and Lemma 2.2 implies that it is even onto $\mathscr{M}$. Since $X^{\prime}$ is standard so is $\mathscr{M}^{\prime}$ while $\mathscr{M}$ is separable since $X$ is separable. We may then conclude that $\mathscr{M}$ is an analytic Borel space.

3. Borel and topological $G$-spaces. Throughout this paper $G$ will denote a fixed locally compact group satisfying the second axiom of countability. We shall regard $G$ as a Borel space by equipping it with the Borel structure generated by the open sets of $G$. $A G$-space will be a Borel space $(X, \mathscr{B})$ together with a map $(g, x) \rightarrow g(x)$ of $G \times X$ into $X$ such that (i) $x \rightarrow g(x)$ is an automorphism of the Borel space $(X, \mathscr{B})$ for each $g$ in $G$, (ii) for all $x \in X$ and $g_{1}, g_{2} \in G, g_{1}\left(g_{2}(x)\right)=$ $=g_{1} g_{2}(x)$. A Borel $G$-space is a $G$-space in which the map $(g, x) \rightarrow g(x)$ is a measurable map of $G \times X$ into $X$. We shall use the same symbol $X$ to denote both the $G$-space and the underlying space on which $G$ acts. A $G$-space is called standard (analytic, etc.) if the underlying Borel space is so. A $G$-space $X$ is said to be topological if (i) $X$ is a completely regular Hausdorff space and the underlying Borel structure $\mathscr{B}$ is the smallest one which makes all continuous functions on $X$ measurable, (ii) the map $(g, x) \rightarrow g(x)$ is continuous from $G \times X$ into $X$. A topological $G$-space is said to be compact (separable, metric, etc.) if the underlying topological space is so.

Let $X$ be any $G$-space. For any set $A \subseteq X$ and any $g$ in $G$ we write $g[A]=\{g(x): x \in A\}$. A set $A$ is said to be invariant if $g[A]=A$ for all $g$. If $\mathscr{B}$ is the underlying Borel structure of $X$ we write $\overline{\mathscr{B}}$ for the $\sigma$-algebra of those sets in $\mathscr{B}$ which are invariant. By an invariant measure we mean a measure $\mu$ for which $\mu(g[A])=\mu(A)$ for all $g$ and $A \in \mathscr{B}$. We write $\mathscr{I}$ for the set of all invariant measures. $\mathscr{I}$ is a convex set. A measure $e$ on $\mathscr{B}$ is said to be ergodic if it is an extreme point of the convex set $\mathscr{I}$. We denote by $\mathscr{E}$ the set of all ergodic measures. We write $\mathscr{N}$ for the $\sigma$-ideal of all sets $A \in \mathscr{B}$ such that $\mu(A)=0$ for every invariant measure $\mu$. We put $\overline{\mathscr{N}}=\mathscr{N} \cap \overline{\mathscr{B}}$. Given an invariant measure $\mu$ and $A \in \mathscr{B}$, 
$A$ is called $\mu$-invariant if $\mu(g[A] \triangle A)=0$ for each $g \in G$. $A \mathscr{B}$-measurable function $f$ on $X$ is said to be $\mu$-invariant if $f\left(g^{-1}(x)\right)=f(x) \mu$-almost everywhere for each $g$ in $G$.

Two $G$-spaces $X$ and $X^{\prime}$ are said to be isomorphic if there exists a map $f: X \rightarrow X^{\prime}$ such that (i) $f$ is an isomorphism between the underlying Borel spaces and (ii) $f(g(x))=g(f(x))$ for all $g$ and $x \in X$. A $G$-space $X$ is said to be a $G$-subspace of a $G$-space $X^{\prime}$ if $X$ is an invariant subspace of $X^{\prime}$ and if the $G$-actions on $X$ and $X^{\prime}$ coincide over $X ; X$ is then said to be the $G$-subspace defined by the invariant subset of $X^{\prime}$ in question.

We shall write $\lambda$ for a fixed left invariant Haar measure on $G$. We denote by $\mathscr{L}^{1}$ the Banach space of (equivalence classes of) $\lambda$-summable Borel functions on $G$, and for any $\phi \in \mathscr{L}^{1}$ we put $\|\phi\|_{1}=\int_{G}|\phi(g)| d \lambda(g)$. Given $\phi, \psi \in \mathscr{L}^{1}$ we write $\phi * \psi$ for the function of $\mathscr{L}^{1}$ defined for almost all $h \in G$ by $(\phi * \psi)(h)=$ $\int_{G} \phi(g) \psi\left(g^{-1} h\right) d \lambda(g)$.

If $X$ is a Borel $G$-space, it follows easily from the measurability of the map $(g, x) \rightarrow g(x)$ that for each bounded $\mathscr{B}$-measurable function $f$ on $X$, the function $x \rightarrow \int_{G} \phi(g) f\left(g^{-1}(x)\right) d \lambda(g)$ is bounded and $\mathscr{B}$-measurable for all $\phi \in \mathscr{L}^{1}$; we denote it by $\phi * f$. We write $\mathscr{F}$ for the Banach space of all bounded $\mathscr{B}$-measurable functions on $X$ and for each $f \in \mathscr{F}$ we put $\|f\|=\sup _{x \in X}|f(x)|$. It is obvious that $\|\phi * f\| \leqq\|\phi\|_{1} \cdot\|f\|$ for all $\phi$ in $\mathscr{L}^{\prime}$ and $f$ in $\mathscr{F}$ and that $f \rightarrow \phi * f$ is a bounded linear operator in $\mathscr{F}$ for each $\phi$.

Unless otherwise stated $X, \mathscr{B}, G, \cdots$ will have the same meaning throughout the rest of the paper and $X$ will be a Borel $G$-space.

Lemma 3.1. Let $X$ be a Borel G-space and $X^{\prime} \subseteq X$ an invariant subset. Then the G-subspace defined by $X^{\prime}$ is a Borel G-space.

Proof. Trivial.

Lemma 3.2. Let $X$ be a topological G-space. Then $X$ is necessarily a Borel G-space.

Proof. We must show that $(g, x) \rightarrow g(x)$ is measurable from $G \times X$ to $X$. Since the Borel structure $\mathscr{B}$ of $X$ is the smallest one that makes all continuous functions on $X$ measurable it is enough to prove that for any continuous $f$ on $X,(g, x) \rightarrow f(g(x))$ is measurable on $G \times X$. Write $u(g, x)=f(g(x))$. It is clear that for each $x$ in $X, g \rightarrow u(g, x)$ is continuous in $g$ while for each $g$ in $G$, $x \rightarrow u(g, x)$ is continuous and hence $\mathscr{B}$-measurable on $X$. Since $G$ is a separable metric space it follows $[14$, p. 286] that $u$ is measurable on $G \times X$.

Lemma 3.3. Let $X$ be a Borel G-space and $A \in \mathscr{B}$. Then there exists an invariant set $A_{0} \in \mathscr{B}$ such that $\mu\left(A \triangle A_{0}\right)=0$ for every invariant measure $\mu$ for which $A$ is $\mu$-invariant. More generally, if $f$ is any $\mathscr{B}$-measurable function there 
exists an invariant $\mathscr{B}$-measurable function $f_{0}$ such that $f=f_{0} \mu$-almost everywhere for any invariant measure $\mu$ for which $f$ is $\mu$-invariant.

Proof. We shall first show how to deduce the second assertion from the first. Given $f$ we can find a sequence $f_{n}$ converging pointwise to $f$ where each $f_{n}$ has the form $\sum_{i} c_{n i} \psi_{A_{n i}}$ where the $c_{n i}$ are constants and the sets $A_{n i}$ are of the type $\left\{x: a_{n i}<f(x) \leqq b_{n i}\right\}$. If we define $f_{n}^{0}=\sum_{i} c_{n i} \psi_{A_{n i}}^{0}$ where $A_{n i}^{0}$ is an invariant set in $\mathscr{B}$ related to $A_{n i}$ as in the lemma, and put $f_{0}(x)=\lim f_{n}^{0}(x)$ for all $x$ for which this limit exists and $f_{0}(x)=0$ for all other $x$, then it is easily verified that $f_{0}$ has all the required properties.

To prove the first assertion, let $A \in \mathscr{B}$ and let $S=\left\{\phi: \phi \geqq 0, \phi \in \mathscr{L}^{1}\right.$, $\left.\|\phi\|_{1}=1\right\}$. For each $\phi \in S$ we define $A_{\phi}=\left\{x:\left(\phi * \psi_{A}\right)(x)=1\right\}$ where $\psi_{A}$ is the characteristic function of $A$. Let $A_{0}=\bigcap_{\phi \in S} A_{\phi}$. Each $A_{\phi}$ is in $\mathscr{B}$ and it follows from the inequality $\|\phi * u\| \leqq\|\phi\|_{1} \cdot\|u\|(u$ in $\mathscr{F})$ that $\bigcap_{\phi \in S} A_{\phi}$ $=\bigcap_{n} A_{\phi_{n}}$ where $\left\{\phi_{1}, \phi_{2}, \cdots\right\}$ is any dense sequence in $S$. Consequently $A_{0} \in \mathscr{B}$. For any $h \in G$ and $\phi \in S$ if we define $\phi^{h}(g)=\phi(h g)$ for all $g$, then it follows from the identity $\left(\phi * \psi_{A}\right)(h(x)) \equiv\left(\phi^{h} * \psi_{A}\right)(x)$ that $x \in A_{\phi^{h}}$ if and only if $h(x) \in A_{\phi}$ and hence we may conclude that $A_{0}$ is invariant. We complete the proof by showing that if $\mu$ is an invariant measure and $A$ is $\mu$-invariant, then $\mu\left(A \Delta A_{0}\right)=0$. It is enough to prove that for all $n \mu\left(A_{\phi . .} \Delta A\right)=0$. If $\mu$ is any invariant measure such that $\mu(g[A] \triangle A)=0$ for all $g$, we deduce that $\psi_{A}\left(g^{-1}(x)\right)=\psi_{A}(x)$ for each $g$ and $\mu$-almost all $x$ and Fubini's theorem enables us to conclude the existence of a set $N \in \mathscr{B}$ such that $\mu(N)=0$ and for each $x$ in $X-N, \quad \psi_{A}\left(g^{-1}(x)\right)=\psi_{A}(x)$ for almost all $g$, so that $\left(\phi * \psi_{A}\right)(x)=\psi_{A}(x)$ for $\mu$-almost all $x$ for each $\phi \in S$. This implies that $\mu\left(A_{\phi_{n}} \Delta A\right)=0$ for all $n$ and hence that $\mu\left(A \triangle A_{0}\right)=0$.

REMARK. It is to be observed that the set $A_{0}$ depends only on $A$ and not on $\mu$.

THEOREM 3.1. Let $X$ be a Borel G-space and $\overline{\mathscr{B}}$ the $\sigma$-algebra of invariant sets in $\mathscr{B}$. Then $\mathscr{E}$ consists precisely of those invariant measures $\mu$ having the property that $\mu(A)=0$ or 1 for each $A \in \overline{\mathscr{B}}$.

Proof. Suppose $\mu \in \mathscr{I}$ and $A \in \overline{\mathscr{B}}$ such that $\mu(A)=c$ where $0<c<1$. If we define measures $\mu_{1}$ and $\mu_{2}$ by setting $\mu_{1}(B)=(1 / c) \mu(B \cap A)$ and $\mu_{2}(B)=(1 /(1-c)$ $\times \mu(B \cap(X-A))$ for all $B \in \mathscr{B}$, then it is obvious that $\mu_{1}, \mu_{2} \in \mathscr{I}, \mu_{1} \neq \mu_{2}$, and $\mu$ $\mu=c \cdot \mu_{1}+(1-c) \cdot \mu_{2}$. This shows that $\mu \notin \mathscr{E}$.

Conversely let $\mu \in \mathscr{I}$ have the property that $\mu(B)=0$ or 1 for each $B \in \overline{\mathscr{B}}$. We shall show that $\mu \in \mathscr{E}$. Suppose in fact that $\mu \notin \mathscr{E}$. Then we may write $\mu=c \cdot \mu_{1}+(1-c) \cdot \mu_{2}$ where $0<c<1, \mu_{1}, \mu_{2} \in \mathscr{I}$ and $\mu_{1} \neq \mu_{2}$. Clearly $\mu_{1}$ and $\mu_{2}$ are absolutely continuous with respect to $\mu$. Let $f_{1}=d \mu_{1} / d \mu$ be the RadonNikodym derivative of $\mu_{1}$ with respect to $\mu$. Since $\mu_{1} \neq \mu$ there exists an $a>0$ such that if $A=\left\{x: f_{1}(x)<a\right\}$, then $0<\mu(A)<1$. Since $\mu_{1}$ and $\mu$ are both invariant it follows easily that for each $g, f_{1}\left(g^{-1}(x)\right)=f_{1}(x)$ for $\mu$-almost all $x$ 
and hence that $\mu(g[A] \triangle A)=0$ for each $g$. By Lemma 3.3 there exists an invariant set $A_{0} \in \mathscr{B}$ such that $\mu\left(A \triangle A_{0}\right)=0$. This implies that $0<\mu\left(A_{0}\right)<1$ and gives us a contradiction.

Our work up to now has been of a preparatory nature. We now begin to examine the relation between topological and Borel $G$-spaces. The classical results of Weil, Mackey and Ambrose-Kakutani $[11 ; 17 ; 2]$ have made it clear that there is an intimate interplay between measure and topology in our context. We shall make the connections much more explicit by our results in this section. In fact we shall spend the remainder of this section in establishing that under mild conditions of a general nature Borel $G$-spaces are isomorphic to topological $G$-spaces.

For any nonempty set $\mathscr{D} \subseteq \mathscr{F}$ we write $Y(\mathscr{D})$ for the set of all functions $\gamma$ on $\mathscr{L}^{1} \times \mathscr{D}$ such that (i) for each $f \in \mathscr{D}, \phi \rightarrow \gamma(\phi, f)$ is linear in $\phi$, (ii) $|\gamma(\phi, f)| \leqq\|\phi\|_{1} \cdot\|f\|$ for all $\phi$ in $\mathscr{L}^{1}$ and $f$ in $\mathscr{D}$. Under the topology of pointwise convergence $Y(\mathscr{D})$ becomes a compact Hausdorff space. The class of Baire subsets of $Y(\mathscr{D})$ is easily seen to coincide with the smallest $\sigma$-algebra of subsets of $Y(\mathscr{D})$ that makes all the maps $\gamma \rightarrow \gamma(\phi, f)$ measurable. The inequality $|\gamma(\phi, f)| \leqq\|\phi\|_{1} \cdot\|f\|$ implies in a straightforward fashion that if $\mathscr{D}$ is separable then $Y(\mathscr{D})$ is even a compact metric space.

LEMMA 3.4. For each $\gamma \in Y(\mathscr{D})$ and $g \in G$ define $g[\gamma](\phi, f)=\gamma\left(\phi^{g}, f\right)$ for all $\phi$ in $\mathscr{L}^{1}$ and $f$ in $\mathscr{D}$ where $\phi^{g}(h)=\phi(g h)$ for all $h$. Then $Y(\mathscr{D})$, together with the map $(g, \gamma) \rightarrow g[\gamma]$, is a compact Hausdorff G-space.

Proof. It is routine verification that $\gamma \rightarrow g[\gamma]$ is a one-to-one map of $Y(\mathscr{D})$ onto itself and that $g_{1} g_{2}[\gamma]=g_{1}\left[g_{2}[\gamma]\right]$ for all $\gamma, g_{1}$ and $g_{2}$. Moreover, if $\gamma, \gamma^{\prime} \in Y(\mathscr{D})$ and $g, g^{\prime} \in G$, the inequality]

$$
\left|\gamma\left(\phi^{g}, f\right)-\gamma^{\prime}\left(\phi^{g^{\prime}}, f\right)\right| \leqq\left|\gamma\left(\phi^{g}, f\right)-\gamma^{\prime}\left(\phi^{g}, f\right)\right|+\left\|\phi^{g}-\phi^{g^{\prime}}\right\|_{1} \cdot\|f\|
$$

shows that the map $(g, \gamma) \rightarrow g[\gamma]$ of $G \times Y(\mathscr{D})$ into $Y(\mathscr{D})$ is continuous. This proves that $Y(\mathscr{D})$ is a compact Hausdorff $G$-space.

THEOREM 3.2. Let $X$ be a standard (respectively analytic) Borel G-space. Then there exists a compact metric G-space $Y$ and an invariant Borel set (respectively analytic set) $X^{\prime} \subseteq Y$ such that the G-space $X$ is isomorphic to the G-subspace of $Y$ defined by $X^{\prime}$.

Proof. Let $A_{1}, A_{2}, \cdots$, be a sequence of sets generating $\mathscr{B}$ and let $\psi_{A_{1}}, \psi_{A_{2}}, \cdots$ be their characteristic functions. Let $\mathscr{D}=\left\{\psi_{A_{1}}, \psi_{A_{2}}, \cdots\right\}$ and form the $G$-space $Y(\mathscr{D})$ described in Lemma 3.4.1We write $Y=Y(\mathscr{D})$. Since $\mathscr{D}$ is countable, $Y$ is a compact metric $G$-space.

For each $x \in X$ write $\gamma_{x}(\phi, f)=(\phi * f)(x)$ for all: $\phi$ in $\mathscr{L}^{1}$ and $f$ in $\mathscr{D}$. Clearly $\gamma_{x} \in Y$ and the map $\xi: x \rightarrow \gamma_{x}$ is a measurable map of $X$ into $Y$. If $g \in G$ the string of 
equations $\gamma_{g(x)}(\phi, f)=(\phi * f)(g(x))=\left(\phi^{g} * f\right)(x)=\gamma_{x}\left(\phi^{g}, f\right)=g\left[\gamma_{x}\right](\phi, f)$ shows that $\xi$ exchanges the $G$-action on $X$ into the $G$-action on $Y$. In particular it follows that $X^{\prime}=\xi[X]$ is an invariant subset of $Y$.

We now claim that $\xi$ is an isomorphism of the $G$-space $X$ with the $G$-subspace defined by $X^{\prime}$. It is enough to prove that $\xi$ is an isomorphism of the Borel space $X$ with the Borel subspace of $Y$ defined by $X^{\prime}$. Since $\xi$ is measurable and $X$ is either standard or analytic it is enough to show that $\xi$ is one-to-one; moreover, once it is proved that $\xi$ is one-to-one, it will also follow that $X^{\prime}$ is analytic or Borel if $X$ is analytic or standard [17, Theorems 3.2 and 4.2]. To prove that $\xi$ is one-to-one let $x, y \in X$ and let $\gamma_{x}=\gamma_{y}$. From the characterization of linear functionals on $\mathscr{L}^{1}$ we infer that $f\left(g^{-1}(x)\right)=f\left(g^{-1}(y)\right)$ for each $f \in \mathscr{D}$ for almost all $g$. Since $\mathscr{D}$ is only countable it follows that for some $g_{0} f\left(g_{0}^{-1}(x)\right)=f\left(g_{0}^{-1}(y)\right)$ for all $f \in \mathscr{D}$. By choice of $\mathscr{D}$ this implies that $g_{0}^{-1}(x)=g_{0}^{-1}(y)$ and hence that $x=y$.

Corollary. Let $X$ be a separable Borel G-space andfor each $x \in X$ let $G_{x}=\{g: g(x)=x\}$. Then each $G_{x}$ is a closed subgroup of $G$.

Proof. The proofs of the facts that $\xi$ is one-to-one and $Y$ is a compact metric $G$-space need only the assumption that $X$ is separable. Since the map $g \rightarrow g[\gamma]$ is continuous for each $\gamma \in Y$, the subgroup $\{g: g[\gamma]=\gamma\}$ is closed for each $\gamma$ and the corollary follows at once.

REMARKS 1. Theorem 3.2 is a generalization of a result of Mackey [17] according to which a subgroup of $G$ is closed if (and only if) the quotient Borel structure of the space of left cosets is separable.

REMARKS 2. It has been pointed out by the referee that Theorem 3.2 is closely related to Lemma 2 of Mackey's paper Point realizations of transformation groups in the Illinois J. Math., Vol. 6, No. 2. Mackey is however concerned with a different question; also the $G$-space in which $X$ is imbedded will be different if one follows his arguments. The writer would like to thank the referee for this and for other suggestions.

If $X$ is not analytic, Theorem 3.2 need not be true. In any case our method fails. The mapping $\xi$ need not be an isomorphism. However, it is obvious that if we replace the Borel structure on $X$ by the smallest one with respect to which all the functions $x \rightarrow(\phi * f)(x)$ are measurable, then $\xi$ is an isomorphism of this new Borel space with the Borel subspace defined by $X^{\prime}$.

We shall now examine the nonanalytic separable case. It will follow as a consequence of our discussion that separable Borel $G$-spaces are, so to speak, essentially isomorphic to separable metric $G$-spaces. The reader who is not interested in this problem may skip the rest of this section and go to $\$ 4$ where Theorem 3.2 is made the basis for obtaining the decomposition of a standard Borel $G$-space into its ergodic parts.

LEMMA 3.5. Let $\mu$ be an invariant measure and let $f \in \mathscr{F}$. Then there exists 
a sequence $\psi_{1}, \psi_{2}, \cdots$ in $\mathscr{L}_{1}$ such that (i) $\psi_{j} \geqq 0,\left\|\psi_{j}\right\|_{1}=1$ for $j=1,2, \cdots$, (ii) $\left\|\psi_{j} * \alpha-\alpha\right\|_{1} \rightarrow 0$ as $j \rightarrow \infty$ for each $\alpha$ in $\mathscr{L}^{1}$, (iii) $\psi_{j} * f \rightarrow f$ as $j \rightarrow \infty \mu$-almost everywhere.

Proof. Let $U_{1}, U_{2}, \cdots$ be a sequence of compact neighbourhoods of the identity in $G$ shrinking to the identity and let $\phi_{n}=\left(1 / \lambda\left(U_{n}\right)\right) \psi_{U_{n}}$. Then $\left\|\phi_{n} * \alpha-\alpha\right\|_{1} \rightarrow 0$ as $n \rightarrow \infty$ for each $\alpha \in \mathscr{L}^{1}[15$, p. 124]. Let $\tau$ be a function on $G$ such that $0<\tau(g)<1$ for all $g$ and $\int_{G} \tau d \lambda<\infty$. For any $x \in X$ write $f_{x}(g)=f(g(x))$. We may assume that $\|f\| \leqq 1$.

For each $x \in X$ the function $\tau \cdot f_{x}$ is in $\mathscr{L}^{1}$ and hence $\left\|\phi_{n} * \tau \cdot f_{x}-\tau \cdot f_{x}\right\|_{1} \rightarrow 0$ as $n \rightarrow \infty$. Moreover, $\left\|\phi_{n} * \tau \cdot f_{x}-\tau \cdot f_{x}\right\|_{1} \leqq 2\|\tau\|_{1}$ and it is easily seen that $x \rightarrow\left\|\phi_{n} * \tau \cdot f_{x}-\tau \cdot f_{x}\right\|_{1}$ is $\mathscr{B}$-measurable. Consequently

$$
\int_{X}\left\|\phi_{n} * \tau \cdot f_{x}-\tau \cdot f_{x}\right\|_{1} d \mu(x) \rightarrow 0
$$

as $n \rightarrow \infty$. This means that there exists a subsequence $n_{1}<n_{2}<\cdots$ such that

$$
\sum_{j} \int_{X}\left\|\phi_{n_{j}} * \tau \cdot f_{x}-\tau \cdot f_{x}\right\|_{1} d \mu(x)<\infty .
$$

Fatou's lemma implies now that

$$
\sum_{j}\left\|\phi_{n_{j}} * \tau \cdot f_{x}-\tau \cdot f_{x}\right\|_{1}<\infty
$$

for $\mu$-almost all $x$. A second use of Fatou's lemma now yields the fact that for $\mu$-almost all $x$

$$
\sum_{j}\left|\phi_{n_{j}} * \tau \cdot f_{x}-\tau \cdot f_{x}\right|<\infty
$$

for almost all $g$. In particular, for $\mu$-almost all $x,\left|\phi_{n_{j}} * \tau \cdot f_{x}-\tau \cdot f_{x}\right| \rightarrow 0$ as $j \rightarrow \infty$ for almost all $g$. By Fubini's theorem it follows that for almost all $g$, $\phi_{n_{j}} * \tau \cdot f_{x} \rightarrow \tau \cdot f_{x}$ for $\mu$-almost all $x$. Since $\left\|\phi_{n_{j}} * \tau-\tau\right\|_{1} \rightarrow 0$ as $j \rightarrow \infty$ also we conclude that for some $g_{0} \in G$ and a further subsequence $m_{1}<m_{2}<\cdots$

$$
\begin{aligned}
\int_{G} \phi_{m_{j}}(g) \tau\left(g^{-1} g_{0}\right) f\left(g^{-1} g_{0}(x)\right) d \lambda(g) & \rightarrow \tau\left(g_{0}\right) f\left(g_{0}(x)\right), \\
\int_{G} \phi_{m_{j}}(g) \tau\left(g^{-1} g_{0}\right) d \lambda(g) & \rightarrow \tau\left(g_{0}\right)
\end{aligned}
$$

hold for $\mu$-almost all $x$. Since $\mu$ is invariant the first relation leads to

$$
\int_{G} \phi_{m_{j}}(g) \tau\left(g^{-1} g_{0}\right) f\left(g^{-1} x\right) d \lambda(g) \rightarrow \tau\left(g_{0}\right) f(x)
$$

for $\mu$-almost all $x$. Write now

and

$$
\begin{gathered}
\beta_{j}(g)=\left(\phi_{m_{j}}(g) \tau\left(g^{-1} g_{0}\right)\right) / \tau\left(g_{0}\right) \\
\psi_{j}=\left(\frac{1}{\left\|\beta_{j}\right\|_{1}}\right) \beta_{j}
\end{gathered}
$$


Clearly $\psi_{j} \geqq 0,\left\|\psi_{j}\right\|_{1}=1$ and $\psi_{j} * f \rightarrow f \mu$-almost everywhere. Since $\psi_{j}$ vanishes outside $U_{n_{j}}$ it follows from a well-known fact $\left[15\right.$, p. 124] that $\left\|\psi_{j} * \alpha-\alpha\right\|_{1} \rightarrow 0$ as $j \rightarrow \infty$ for all $\alpha$ in $\mathscr{L}^{1}$. The lemma is proved.

For any subset $\mathscr{D}$ of $\mathscr{F}$ we write $\hat{\mathscr{B}}(\mathscr{D})$ for the smallest $\sigma$-algebra of subsets of $X$ which makes the functions $x \rightarrow(\phi * f)(x)$ measurable for all $\phi$ in $\mathscr{L}^{1}$ and $f$ in $\mathscr{D}$. For any $g$ in $G,(\phi * f)(g(x))=\left(\phi^{g} * f\right)(x)$, which shows that $x \rightarrow g(x)$ is an automorphism of the Borel space $(X, \hat{\mathscr{B}}(\mathscr{D}))$. This Borel space, which we denote by $\hat{X}(\mathscr{D})$, is thus the underlying space of a $G$-space. We shall denote this $G$-space also by $\hat{X}(\mathscr{D})$.

LEMMA 3.6. $\hat{X}(\mathscr{D})$ is a Borel G-space for any $\mathscr{D} \subseteq \mathscr{F}$. If $\mathscr{D}$ is separable under the norm of $\mathscr{F}$ and separates the points of $X$, then $\hat{X}(\mathscr{D})$ is isomorphic to the G-subspace of a compact metric G-space.

Proof. To prove that $\hat{X}(\mathscr{D})$ is a Borel $G$-space we must show that the map $(g, x) \rightarrow g(x)$ is a measurable map of $G \times \hat{X}(\mathscr{D})$ into $\hat{X}(\mathscr{D})$. This will be shown if we show that the map $(g, x) \rightarrow(\phi * f)(g(x))=\left(\phi^{g} * f\right)(x)$ is measurable on $G \times \hat{X}(\mathscr{D})$. Now for each $x$ in $X$, the function $g \rightarrow\left(\phi^{g} * f\right)(x)$ is continuous on the separable metric space $G$ while the function $x \rightarrow\left(\phi^{g} * f\right)(x)$ is $\hat{\mathscr{B}}(\mathscr{D})$-measurable for each fixed $g$ in $G$. By a well-known theorem $[14$, p. 286] it follows that $(g, x) \rightarrow(\phi * f)(g(x))$ is measurable on $G \times \hat{X}(\mathscr{D})$.

When $\mathscr{D}$ is separable and separates the points of $X$, the argument given in the proof of Theorem 3.2 shows that $\xi$ is an imbedding of $\hat{X}(\mathscr{D})$ as a $G$-subspace of $Y\left(\mathscr{D}_{0}\right)$ where $\mathscr{D}_{0}$ is a denumerable dense subset of $\mathscr{D}$. The lemma is proved.

LEMMA 3.7. Suppose that $\mathscr{B}_{1}$ is the smallest $\sigma$-algebra of subsets of $X$ with respect to which all functions of $\mathscr{D}$ are measurable. If $\mu$ is any invariant measure then $\mathscr{B}_{1} \subseteq \hat{\mathscr{B}}(\mathscr{D})_{\mu}$ where $\hat{\mathscr{B}}(\mathscr{D})_{\mu}$ denotes the $\mu$-completion of the $\sigma$-algebra $\hat{\mathscr{B}}(\mathscr{D})$.

Proof. It is enough to prove that each $f \in \mathscr{D}$ is $\hat{\mathscr{B}}(\mathscr{D})_{\mu}$-measurable. Now by Lemma 3.3 there is a sequence $\psi_{1}, \psi_{2}, \cdots$ in $\mathscr{L}^{1}$ such that $\psi_{j} * f \rightarrow f \mu$-almost everywhere. Since each $\psi_{j} * f$ is $\mathscr{\mathscr { B }}(\mathscr{D})$-measurable it follows at once that $f$ is $\hat{\mathscr{B}}(\mathscr{D})_{\mu}$-measurable.

THEOREM 3.3. Suppose $X$ is a Borel G-space and $\mu$ an invariant measure on $X$. Suppose further that there is a countably generated $\sigma$-algebra $\mathscr{B}^{1}$ such that $\mathscr{B}^{1}$ separates the points of $X$ and $\mathscr{B}$ is contained in the $\mu$-completion of $\mathscr{B}^{1}$. Then there exists a separable metric topology over $X$ with the class $\mathscr{B}^{\prime}$ of Borel sets such that (i) $X$, equipped with this topology, is a topological G-space, (ii) $\mathscr{B}^{\prime} \subseteq \mathscr{B} \subseteq \mathscr{B}_{\mu}^{\prime}$ where $\mathscr{B}_{\mu}^{\prime}$ is the $\mu$-completion of $\mathscr{B}^{\prime}$.

Proof. Let $A_{1}, A_{2}, \cdots$ be a sequence of sets separating points of $X$ and generating $\mathscr{B}^{1}$. Let $\mathscr{D}$ be the set $\left\{\psi_{A_{1}}, \psi_{A_{2}}, \cdots\right\}$. The imbedding procedure outlined in Theorem 3.2 can be used in an analogous fashion to prove that the Borel 
$G$-space $X$ (equipped with $\hat{\mathscr{B}}(\mathscr{D})$ ) is isomorphic to a subspace of a compact metric $G$-space. In other words, if we write $\mathscr{B}^{\prime}=\hat{\mathscr{B}}(\mathscr{D})$, there is a separable metric topology for $X$ under which $\mathscr{B}^{\prime}$ becomes the class of Borel subsets of $X$, and equipped with which $X$ becomes a topological $G$-space. Lemma 3.5 now implies that $\mathscr{B}^{\prime} \subseteq \mathscr{B} \subseteq \mathscr{B}_{\mu}^{\prime}$ and completes the proof.

REMARK. Theorem 3.3 is a generalization of a theorem of Ambrose and Kakutani [2]. It may be observed that we have proved a slightly sharper result than described in the statement of the theorem. Indeed the separable metric $G$-space $X$ is even isomorphic to a $G$-subspace of a compact metric $G$-space.

As a final result we shall obtain conditions under which a Borel $G$-space is isomorphic to a $G$-subspace of a compact Hausdorff-space. We write $\hat{\mathscr{B}}$ for the smallest $\sigma$-algebra of subsets of $X$ that makes all the maps $x \rightarrow(\phi * f)(x)$ measurable $\left(\phi\right.$ in $\mathscr{L}^{1}, f$ in $\left.\mathscr{F}\right) . \hat{\mathscr{B}}=\hat{\mathscr{B}}(\mathscr{F})$ in the notation of Lemma 3.4. We shall say that $X$ is separated if given $x, y \in X$ and $x \neq y$, there is an $A \in \mathscr{B}$ such that $x \in A$ and $y \in X-A$.

LEMMA 3.8. If $X$ is a compact Hausdorff $G$-space, then $\hat{\mathscr{B}}=\mathscr{B}$.

Proof. Let $C$ be the closed linear subspace of $\mathscr{F}$ consisting of all continuous functions on $X$ and for any $f \in C$ and $g$ in $G$ write $\left(W_{g} f\right)(x)=f\left(g^{-1}(x)\right)$. For any $f$ in $C$ and $\phi$ in $\mathscr{L}^{1}$ the function $x \rightarrow(\phi * f)(x)$ is continuous. Let $D$ denote the set of all such functions as $\phi$ varies over $\mathscr{L}^{1}$ and $f$ over $C$.

We claim that $D$ separates the points of $X$. Suppose in fact that $x, y \in X$ and $x \neq y$. Then there is an $f_{0} \in C$ for which $f_{0}(x) \neq f_{0}(y)$. Let $L$ be the closed linear subspace spanned by all the $W_{g} f_{0}$ as $g$ varies in $G$. Since $X$ is compact and $G$ acts continuously on $X, L$ is separable. Suppose now that $D$ does not separate $x$ and $y$. Then for each $u \in L,(\phi * u)(x)=(\phi * u)(y)$ for all $\phi$ in $\mathscr{L}^{1}$ and hence $u\left(g^{-1}(x)\right)=u\left(g^{-1}(y)\right)$ for almost all $g$. Since $L$ is separable there will be some $g_{0}$ such that $u\left(g_{0}^{-1}(x)\right)=u\left(g_{0}^{-1}(y)\right)$ for all $u \in L$. If we now define $u\left(x^{\prime}\right)$ $=f_{0}\left(g_{0}\left(x^{\prime}\right)\right)$ for all $x^{\prime} \in X$ then $u \in L$ and we can infer that $f_{0}(x)=f_{0}(y)$, a contradiction. $D$ thus separates the points of $X$.

By the Stone-Weierstrass theorem the algebra generated by $D$ is dense in $C$ and since each element of $D$ is $\hat{\mathscr{B}}$-measurable we reach the conclusion that every $f$ in $C$ is $\hat{\mathscr{B}}$-measurable. This proves that $\hat{\mathscr{B}}$ coincides with the class of Baire sets of $X$, i.e., $\hat{\mathscr{B}}=\mathscr{B}$.

THEOREM 3.4. A set of necessary and sufficient conditions that a Borel Gspace $X$ be isomorphic to a G-subspace of a compact Hausdorff G-space is that $X$ be separated and $\hat{\mathscr{B}}=\mathscr{B}$.

Proof. Let $Y=Y(\mathscr{F})$ be the compact Hausdorff $G$-space described in Lemma 3.2 and for each $x$ in $X$ let $\gamma_{x}(\phi, f)=(\phi * f)(x)$. Then $\gamma_{x} \in Y$ and $\xi\left(x \rightarrow \gamma_{x}\right)$ is a measurable map of $X$ into $Y$ with $\gamma_{g(x)}=g\left[\gamma_{x}\right]$ for all $g$ and $x$. Write $X^{\prime}=\xi[X]$. $X^{\prime}$ is an invariant subset of $Y$. 
Suppose now that $X$ is separated and $\hat{\mathscr{B}}=\mathscr{B}$. The fact that $X$ is separated implies that $\mathscr{B}$ separates points of $X$. Since $\hat{\mathscr{B}}=\mathscr{B}$ it follows that $\hat{\mathscr{B}}$ separates points of $X$. This implies that the family of functions $x \rightarrow \gamma_{x}(\phi, f)$ separates points of $X$ and hence that $\xi$ is one-to-one. Consequently, $\xi$ is an isomorphism of the Borel space $(X, \hat{\mathscr{B}})$ with the Borel subspace of $Y$ defined by $X^{\prime}$. Since $\hat{\mathscr{B}}=\mathscr{B}$ it follows that $\xi$ is an isomorphism of $X$ and $X^{\prime}$.

We now prove the converse. Suppose that $Y$ is a compact Hausdorff $G$-space and $X$ an invariant subset of $Y$. The Borel subspace defined by $X$ is clearly separated. We now show that $\widehat{\mathscr{B}}=\mathscr{B}$. Let $\mathscr{B}_{Y}$ denote the class of Baire subsets of $Y$ and $\hat{\mathscr{B}}_{Y}$ the smallest $\sigma$-algebra of subsets of $Y$ that makes all the maps $y \rightarrow\left(\phi * f^{\prime}\right)(y)$ measurable ( $\phi$ in $\mathscr{L}^{1}$ and $f^{\prime}$ bounded $\mathscr{B}_{Y}$-measurable). By Lemma $3.8 \mathscr{B}_{Y}=\mathscr{\mathscr { B }}_{Y}$. Since $\mathscr{B}=\mathscr{B}_{Y} \cap X$ it is thus enough to prove that $\widehat{\mathscr{B}}=\widehat{\mathscr{B}}_{Y} \cap X$. This, however, follows from the fact that given any bounded $\mathscr{B}$-measurable $f$ there exists a bounded $\mathscr{B}_{Y}$-measurable $f^{\prime}$ such that $f$ and $\phi * f$ are the restrictions to $X$ of $f^{\prime}$ and and $\phi * f^{\prime}$ respectively.

The theorem is proved.

COROLlaRY. If $G$ is countable and $X$ is a separated $G$-space, $X$ is isomorphic to a G-subspace of a compact Hausdorff G-space.

Proof. If $\delta$ is the function equal to 1 at the identity of $G$ and 0 elsewhere, it is clear that $\delta * f=f$ for all $f$ in $\mathscr{F}$. This implies that $\hat{\mathscr{B}}=\mathscr{B}$ and proves the corollary.

4. Decomposition into ergodic parts. Our aim in this section is to prove that any standard Borel $G$-space can be decomposed in a "measurable fashion" into disjoint invariant Borel sets on each of which the group acts uniquely ergodically.

Suppose $X$ is a Borel $G$-space and $\mathscr{E}$ the space of ergodic measures. Equipped with what we have called the customary Borel structure $\left({ }^{2}\right)$ (in $\S 2$ ), $\mathscr{E}$ is a Borel space and it makes sense to talk of measurable mappings into $\mathscr{E}$. Suppose $\mathscr{E}$ is nonempty. A map $\beta\left(x \rightarrow \beta_{x}\right)$ of $X$ into $\mathscr{E}$ is said to be a decomposition map of the $G$-space $X$ if it satisfies the following conditions: (i) $\beta$ is a measurable map of $X$ into $\mathscr{E}$, (ii) $\beta$ is invariant, i.e., $\beta_{g(x)}=\beta_{x}$ for all $g$ in $G$ and $x$ in $X$, (iii) If $X_{e}=\left\{x: \beta_{x}=e\right\}$ for $e$ in $\mathscr{E}$, then $X_{e} \in \mathscr{B}, e\left(X_{e}\right)=1$ for all $e$, and for any invariant measure $\mu$,

$$
\mu(A)=\int_{X} \beta_{x}\left(A \cap X_{\beta_{x}}\right) d \mu(x)
$$

for all $A \in \mathscr{B}$.

Some remarks are in order concerning the above definition. In the first place, since $\beta_{x}\left(X_{\beta_{x}}\right)=1$ for all $x$, the integrand in (D) is just $\beta_{x}(A)$. Since $\beta$ is a measurable map of $X$ into $\mathscr{E}$ it follows that $x \rightarrow \beta_{x}(A)$ is measurable for each $A$ in $\mathscr{B}$. Moreover

(2) We shall use the symbol $\mathscr{A}$ for this particular Borel structure. 
$0 \leqq \beta_{x}(A) \leqq 1$ for all $x$ in $X$ and $A$ in $\mathscr{B}$ so that the integrals in (D) make sense. Secondly it may be noted that each $X_{e}$ is an invariant set and will automatically belong to $\mathscr{B}$ as soon as each single element set in $\mathscr{E}$ belongs to its Borel structure. Our final remark can be described in the following lemma.

LeMma 4.1. Let $\beta$ be a decomposition map of the Borel G-space, let $\mathscr{E} \neq \varnothing$, and let $E$ be a subset of $\mathscr{E}$ belonging to the Borel structure of $\mathscr{E}\left({ }^{2}\right)$. If $A=\beta^{-1}(E)$ then $\beta_{x}(A)=0$ or 1 according as $x \notin A$ or $x \in A$.

Proof. The proof follows from the fact that $\beta_{x}\left(X_{\beta_{n}}\right)=1$ for all $x$.

Given two decomposition maps $\beta, \beta^{\prime}$ of a Borel $G$-space $X$ we shall say that $\beta$ and $\beta^{\prime}$ are essentially equal if there exist a set $N \in \mathscr{N}$ and $\beta_{x}=\beta_{x}^{\prime}$ for all $x \in X-N$. Notice that if $\beta$ is a decomposition map of $X$ and $N \in \mathscr{N}$ then $\beta$ maps $X-N$ onto $\mathscr{E} ;$ indeed since $e(X-N)=e\left(X_{e}\right)=1$ for any $e$ in $\mathscr{E}, X_{e} \cap X-N$ is clearly non-null. We also introduce the notion of unique ergodicity. We shall say that a Borel $G$-space $X$ is uniquely ergodic if there exists a measure $\mu$ on $X$ such that $\mathscr{I}=\mathscr{E}=\{\mu\}$. Applying formula (D) to subsets of $X_{e}$ we easily see that the $G$-subspace defined by each $X_{e}$ is uniquely ergodic with $e$ as the unique invariant measure.

The main theorem of this section asserts that every standard Borel $G$-space admits an essentially unique decomposition map. We now proceed to develop the auxiliary propositions needed to establish this result.

LeMma 4.2. Let $\mathscr{E} \neq \varnothing$ and let $\beta$ be a decomposition map of $X$. If $A$ is any invariant set $\mathscr{B}$ there exists a set $E \subseteq \mathscr{E}$ such that $E \in \mathscr{A}$ and $A \triangle \beta^{-1}(E) \in \mathscr{N}$. More precisely, if $E=\{e: e \in \mathscr{E}, e(A)=1\}$, then $\mu\left(A \Delta \beta^{-1}(E)\right)=0$ for all $\mu \in \mathscr{I}$.

Proof. Write $B=\beta^{-1}(E)$. We must show that $A \triangle B=(A-B) \cup(B-A)$ is in $\mathscr{N}$. Since we may argue with $X-A, X-B$ and $\mathscr{E}-E$ instead of $A, B$ and $E$ it is enough to show that $B-A$ is in $\mathscr{N}$. Suppose $\mu$ is an invariant measure. By the definition of $B, \beta_{x}(B-A) \leqq \beta_{x}(X-A)=0$ for $x$ in $B$ while by Lemma 4.1, $\beta_{x}(B-A) \leqq \beta_{x}(B)=0$ for $x$ outside $B$. Consequently $\mu(B-A)=\int_{X} \beta_{x}(B-A) d \mu(x)=0$. The lemma is proved.

Let $\beta$ be a decomposition map and for each $f \in \mathscr{F}$ define $f^{*}$ by setting $f^{*}(x)=\int_{X} f(y) d \beta_{x}(y)$ for all $x$ in $X$. Let $U$ be the mapping $f \rightarrow f^{*}$.

LEMMA 4.3. U has the following properties: (i) Uf is $\overline{\mathscr{B}}$-measurable for each $f$ in $\mathscr{F}$ and $U f=f$ if $f$ is a constant, (ii) $\|U f\| \leqq\|f\|$ for all $f$ in $\mathscr{F}$, (iii) for each invariant measure $\mu$ and each invariant $A$ in $\mathscr{B}$, we have $\int_{A} f d \mu=\int_{A}(U f) d \mu$.

Proof. Clearly $U$ is linear and $\|U f\| \leqq\|f\|$ for all $f$ in $\mathscr{F}$. Moreover, for any set $A$ in $\mathscr{B}, U \psi_{A}$ is $\overline{\mathscr{B}}$-measurable. It follows then easily that $U f$ is $\overline{\mathscr{B}}$-measurable for each $f$ in $\mathscr{F}$. It therefore remains to prove (iii). Suppose $A=\beta^{-1}(E)$ where $E \subseteq \mathscr{E}$ and $E \in \mathscr{A}$. For any set $B$ in $\mathscr{B}$ we have 


$$
\begin{aligned}
\int_{\boldsymbol{A}}\left(U \psi_{B}\right) d \mu & =\int_{\boldsymbol{A}} \beta_{x}(B) d \mu(x) \\
& =\int_{X} \beta_{x}(B \cap A) d \mu(x)
\end{aligned}
$$

since for $x \in A, \beta_{x}(A)=1$ while for $x \notin A, \beta_{x}(A)=0$; and this last integral is simply $\mu(B \cap A)=\int_{A} \psi_{B} d \mu$. In other words (iii) is true whenever $f=\psi_{B}$ and hence for any $f$ in $\mathscr{F}$. Using Lemma 4.2 we now infer (iii) for all $f$ in $\mathscr{F}$ and $A$ in $\overline{\mathscr{B}}$.

Lemma 4.3 points up to a key property of decomposition maps. The property (iii) implies that for any $f$ in $\mathscr{F}$ and any invariant $\mu, U f$ is the so-called conditional expectation of $f$ given the $\sigma$-algebra $\overline{\mathscr{B}}$, denoted by $E_{\mu}(f \mid \overline{\mathscr{B}})[9 ; 16]$. Notice that given $f$ and $\mu$, (iii) determines $U f \mu$-almost everywhere. We can now prove the essential uniqueness of the decomposition maps.

LEMMA 4.4. If $\beta$ and $\beta^{\prime}$ are two decomposition maps of a separable Borel $G$-space then $\beta$ and $\beta^{\prime}$ are essentially equal.

Proof. Let $U$ and $U^{\prime}$ be the mappings associated with $\beta$ and $\beta^{\prime}$ respectively, having the properties described in Lemma 4.3. Since $\left(U \psi_{A}\right)(x)=\beta_{x}(A)$ and $\left(U^{\prime} \psi_{A}\right)(x)=\beta_{x}^{\prime}(A)$ and since conditional expectations are essentially uniquely determined, it follows that if we set $N_{A}=\left\{x: \beta_{x}(A) \neq \beta_{x}^{\prime}(A)\right\}$, then $N_{A} \in \overline{\mathscr{B}}$ and $\mu\left(N_{A}\right)=0$ for each invariant measure $\mu$. Let $\left\{A_{1}, A_{2}, \cdots\right\}$ be a sequence of sets forming a Boolean algebra generating $\mathscr{B}$. If we define $N=\bigcup_{n} N_{A_{n}}$ then $\mu(N)=0$ for each $\mu$ in $\mathscr{I}$ and $\beta_{x}=\beta_{x}^{\prime}$ for $x$ in $X-N$.

Our problem now is the construction of decomposition maps and the first step in our solution is the construction of a map $U$ having the properties described in Lemma 4.3. To motivate our approach to the construction of $U$ let us consider the special case of a $G$-space where $G$ is the group of all integers $\{0, \pm 1, \pm 2, \cdots\}$, i.e., when we have the group of iterates of a single automorphism $\tau$ of $(X, \mathscr{B})$. In this case if we define, for any bounded $\mathscr{B}$-measurable $f$,

$$
(U f)(x)=\left\{\begin{array}{l}
\lim _{n \rightarrow \infty} 1 / n\left(f(x)+f(\tau(x))+\cdots+f\left(\tau^{n-1}(x)\right)\right) \text { if this limit exists, } \\
0 \quad \text { otherwise }
\end{array}\right.
$$

then the individual ergodic theorem of Birkhoff leads us to the conclusion that the mapping $f \rightarrow U f$ has all the properties described in Lemma $4.3\left({ }^{3}\right)$. Part of the difficulty of the problem in the general case of an arbitrary $G$ is the absence of ergodic theorems of the Birkhoff type.

(3) Note that the $U$ defined here is not linear while that in Lemma 4.3 is linear. 
The next proposition asserts the existence of such mappings in the general case.

THEOREM 4.1. Let $X$ be any Borel G-space. Then there exists a mapping $U$ of $\mathscr{F}$ into $\mathscr{F}$ with the following properties: (i) Uf is $\overline{\mathscr{B}}$-measurable for each $f$ in $\mathscr{F}$ and $U f=f$ whenever $f$ is constant, (ii) $\|U f\| \leqq\|f\|$ for all $f$, (iii) for each invariant measure $\mu$ and $A$ in $\overline{\mathscr{B}}, \int_{A} f d \mu=\int_{A}(U f) d \mu$.

Moreover, if $U$ and $U^{\prime}$ are two such mappings, then for any $f, U f=U^{\prime} f \mu$ almost everywhere for each invariant measure $\mu$, i.e., $\left\{x:(U f)(x) \neq\left(U^{\prime} f\right)(x)\right\} \in \overline{\mathscr{N}}$.

Proof. Let $G_{0}=\left\{g_{1}, g_{2}, \cdots\right\}$ be a countable dense subgroup of $G$. Write $G_{k}$ for the subgroup generated by $\left\{g_{1}, \cdots, g_{k}\right\}$, and $Z_{k}$ for the subgroup generated by $g_{k}$. Clearly $G_{1} \subseteq G_{2} \subseteq \cdots, \bigcup_{k=1}^{\infty} G_{k}=G_{0}$ and $G_{k+1}$ is the subgroup generated by $G_{k}$ and $Z_{k+1}$. Obviously $Z_{1}=G_{1}$. For any subgroup $H \subseteq G_{0}$ we write $\overline{\mathscr{B}}_{H}$ for the class of all sets $A$ in $\mathscr{B}$ which are invariant under the action of the elements of $H$. Clearly $\overline{\mathscr{B}}_{G_{1}} \supseteq \overline{\mathscr{B}}_{G_{2}} \supseteq \cdots, \overline{\mathscr{B}}_{G_{k+1}}=\overline{\mathscr{B}}_{G_{k}} \cap \overline{\mathscr{B}}_{Z_{k+1}}$ and $\bigcap_{k=1}^{\infty} \overline{\mathscr{B}}_{G_{k}}=\overline{\mathscr{B}}_{G_{0}}$. Finally, for any subgroup $H \subseteq G_{0}$ we write $\mathscr{U}_{H}$ for the class of all mappings $U_{H}$ of $\mathscr{F}$ into $\mathscr{F}$ such that (i) for each $f$ in $\mathscr{F}, U_{H} f$ is $\overline{\mathscr{B}}_{H}$-measurable and $U_{H} f=f$ when $f$ is a constant, (ii) $\left\|U_{H} f\right\| \leqq\|f\|$ for all $f$, (iii) for any invariant measure $\mu$ and any $A$ in $\overline{\mathscr{B}}_{H}, \int_{A} f d \mu=\int_{A}\left(U_{H} f\right) d \mu$.

We shall first prove by induction that the classes $\mathscr{U}_{G_{1}}, \mathscr{U}_{G_{2}}, \cdots$ are all nonempty. Our motivating remarks already imply that $\mathscr{U}_{H}$ is nonempty whenever $H$ has a single generator. Suppose $\mathscr{U}_{G_{l}}$ is nonempty for $1 \leqq l \leqq k-1$. Choose an arbitrary mapping $U_{k-1}$ from $\mathscr{U}_{G_{k-1}}$ and a mapping $V$ from $\mathscr{U}_{\mathbf{Z}_{k}}$. We now define mappings $W_{1}, W_{2}, \cdots$ in succession by setting $W_{1}=U_{k-1}, W_{2}=V U_{k-1}, W_{3}=U_{k-1} V U_{k-1}, \cdots$ and so on. Write, for any $f$ in $\mathscr{F}, N_{f}=\left\{x: \lim _{n \rightarrow \infty}\left(W_{n} f\right)(x)\right.$ exists $\}$ and $\bar{N}_{f}$ $=\bigcap_{g \in G_{k}} g\left[N_{f}\right]$. Put

$$
\left(U_{k} f\right)(x)= \begin{cases}\lim _{n \rightarrow \infty}\left(W_{n} f\right)(x) & \text { if } x \in \bar{N}_{f} \\ 0 & \text { otherwise }\end{cases}
$$

$U_{k}$ is a well-defined map of $\mathscr{F}$ into itself and $\left\|U_{k} f\right\| \leqq\|f\|$. It is also easily shown that $U_{k} f$ is $\overline{\mathscr{B}}_{G_{k}}$-measurable for each $f$ in $\mathscr{F}$ and $U_{k} f=f$ if $f$ is a constant. Suppose now $\mu$ is any invariant measure and $f$ any function in $\mathscr{F}$. Since $V u=E_{\mu}\left(u \mid \overline{\mathscr{B}}_{Z_{k}}\right)$ and $U_{k-1} u=E_{\mu}\left(u \mid \overline{\mathscr{B}}_{G_{k-1}}\right)$ for all $u$ in $\mathscr{F}$ it follows from a result of BurkholderChow [6] that $\lim _{n \rightarrow \infty}\left(W_{n} f\right)(x)$ exists for $\mu$-almost all $x$. This means that $\mu\left(X-N_{f}\right)=0$ and hence $\mu\left(X-\bar{N}_{f}\right)=0$. If now $u$ is any element in $\mathscr{F}$ and $A \in \overline{\mathscr{B}}_{G_{k}}$ it follows that $\int_{A} u d \mu=\int_{A} V u d \mu=\int_{A}\left(U_{k-1} u\right) d \mu$. Consequently we have $\int_{A} f d \mu=\int_{A}\left(W_{1} f\right) d \mu=\int_{A}\left(W_{2} f\right) d \mu=\cdots$. We may now conclude that $\int_{A} f d \mu=\int_{A}\left(U_{k} f\right) d \mu$. This proves that $U_{k} \in \mathscr{U}_{G_{k}}$ and hence that $\mathscr{U}_{G_{k}}$ is nonempty. By induction we infer that all the $\mathscr{U}_{G}$, are nonempty for $l=1,2, \cdots$. 
We now show that $\mathrm{U}_{G_{0}}$ is also nonempty. Let $U_{k}$ be an arbitrary element of $\mathscr{U}_{G_{k}}$ for $k=1,2, \cdots$. For $f$ in $\mathscr{F}$ define $M_{f}=\left\{x: \lim _{k \rightarrow \infty}\left(U_{k} f\right)(x)\right.$ exists $\}$ and $\bar{M}_{f}=\bigcap_{g \in G_{0}} g\left[M_{f}\right]$. Put

$$
\left(U_{0} f\right)(x)= \begin{cases}\lim _{k \rightarrow \infty}\left(U_{k} f\right)(x) & \text { if } x \in \bar{M}_{f} \\ 0 & \text { otherwise }\end{cases}
$$

$U_{0}$ is a mapping of $\mathscr{F}$ into $\mathscr{F}$ and $\left\|U_{0} f\right\| \leqq\|f\|$ for all $f$. Moreover, it is easily seen that $U_{0} f$ is $\overline{\mathscr{B}}_{G_{0}}$-measurable for all $f$ and $U_{0} f=f$ if $f$ is a constant. Suppose now $\mu$ is any invariant measure. Since $U_{k} f=E_{\mu}\left(f \mid \overline{\mathscr{B}}_{G_{k}}\right)$ and since $\overline{\mathscr{B}}_{G_{1}} \supseteq \overline{\mathscr{B}}_{G_{2}} \supseteq \cdots$ it follows from the so-called martingale-reversed-sequence convergence theorem that $\lim _{k \rightarrow \infty}\left(U_{k} f\right)(x)$ exists for $\mu$-almost all $x[9 ; 16]$ i.e., $\mu\left(X-M_{f}\right)=0$ and hence $\mu\left(X-\bar{M}_{f}\right)=0$. If now $A \in \overline{\mathscr{B}}_{G_{0}}$, then $A$ is in all, the $\overline{\mathscr{B}}_{G_{k}}$ and hence $\int_{A} f d \mu=\int_{A}\left(U_{k} f\right) d \mu$ for all $k$. This implies that $\int_{A} f d \mu=\int_{A}\left(U_{0} f\right) d \mu$ and shows that $U_{0}$ is in the class $\mathscr{U}_{G_{0}}$.

For any $f$ in $\mathscr{F}, U_{0} f$ is invariant under the action of all the elements of $G_{0}$. We now claim that $U_{0} f$ is $\mu$-invariant for each invariant measure $\mu$. In fact, if for $g$ in $G$ and $u$ in $\mathscr{L}^{2}(\mu)$ we write $\left(L_{g} u\right)(x)=u\left(g^{-1} x\right)$, then $g \rightarrow L_{g}$ is a continuous $\left(^{4}\right)$ unitary representation of $G$ in $\mathscr{L}^{2}(\mu)$; in particular, if $h_{n} \rightarrow h$ then $\int_{X}\left|L_{h_{.}} u-L_{h} u\right|^{2} d \mu \rightarrow 0$. Since $L_{g_{r}}\left(U_{0} f\right)=U_{0} f$ for all $r=1,2, \cdots$ and since $G_{0}$ is dense in $G$, it follows that for each $g$ in $G, L_{g}\left(U_{0} f\right)=U_{0} f \mu$-almost everywhere.

By Lemma 3.3 we can find, corresponding to each $f$ in $\mathscr{F}$, an invariant $\mathscr{B}$ measurable function $f^{*}$ such that $U_{0} f=f^{*} \mu$-almost everywhere for each invariant measure $\mu$. By changing $f^{*}$ on invariant sets in $\mathscr{N}$ we can even ensure that $\left\|f^{*}\right\| \leqq\|f\|$ for all $f$ and that $f^{*}=f$ whenever $f$ is a constant.

If we now define $U f=f^{*}$ for each $f$ then $U$ has all the properties demanded in Theorem 4.1.

The essential uniqueness of conditional expectations implies that if $U^{\prime}$ is another such mapping, then for each $f$ in $\mathscr{F}$ and $\mu$ in $\mathscr{I}, U f=U^{\prime} f \mu$-almost everywhere.

This proves the theorem.

REMARK. If $f$ is in $\mathscr{F}$ and we define $\left(L_{g} f\right)(x)=f\left(g^{-1}(x)\right)$ then $U\left(L_{g} f\right)=U f \mu$ almost everywhere for each $\mu$ in $\mathscr{I}$. In fact, since $\mu$ is invariant, $\int_{A} U\left(L_{g} f\right) d \mu$ $=\int_{A}\left(L_{g} f\right) d \mu=\int_{A} f d \mu=\int_{A}(U f) d \mu$ for any invariant $A$ in $\mathscr{B}$ so that the first and the last equations imply that $U\left(L_{g} f\right)=U f \mu$-almost everywhere. We thus have $\left\{x:\left(U L_{g} f\right)(x) \neq(U f)(x)\right\} \in \mathscr{N}$.

We need two technical lemmas before proving the decomposition theorem. Let $Z$ be a normal Hausdorff space and $\mathscr{S}$ the associated Borel structure. Let $\Gamma$ be

(4) The continuity follows from the measurability of $g \rightarrow \int_{X} v \cdot L_{g} u \cdot d \mu$ for each $u$ and $v$ in $\mathscr{L}^{2}(\mu)$; cf. [15]. 
a nonempty convex set of measures on $\mathscr{B}$ which is compact in its weak*-topology and let $\Gamma^{\prime}$ be the set of extreme points of $\Gamma$. $\Gamma^{\prime}$ is nonempty by the Krein-Mil'man theorem.

Lemma 4.5. For any $A \in \mathscr{B}, \mu(A)=0$ for all $\mu$ in $\Gamma^{\prime}$ implies that $\mu(A)=0$ for all $\mu$ in $\Gamma$.

Proof. It is known [22] that for any measure $v$ on $\mathscr{B}, v(A)=\sup \{v(C): C \subseteq A$, $C$ a closed $\left.G_{\delta}\right\}$. We may therefore assume that $A$ itself is a closed $G_{\delta}$. Write $c=\sup \{\mu(A): \mu \in \Gamma\}$ and $\Gamma_{1}=\{\mu: \mu \in \Gamma, \mu(A)=c\}$.

We claim that $\Gamma_{1}$ is a compact, convex nonempty subset of $\Gamma$. In fact let $\left\{\mu_{\alpha}\right\}$ be a net in $\Gamma$ such that $\mu_{\alpha}(A) \rightarrow c$. Since $\Gamma$ is compact we may assume that $\mu_{\alpha} \Rightarrow v$ for some $v$ in $\Gamma, \Rightarrow$ denoting weak*-convergence. Now $v(A) \geqq \lim _{\alpha} \sup \mu_{\alpha}(A)$ since $A$ is a closed $G_{\delta}[22]$ so that $v(A) \geqq c$, showing that $v$ is in $\Gamma_{1}$. A similar argument leads to the conclusion that $\Gamma_{1}$ is closed, hence compact. The convexity of $\Gamma_{1}$ is obvious.

Let $\mu^{\prime}$ be an extreme point of $\Gamma_{1}$. Such a $\mu^{\prime}$ exists by the Krein-Mil'man theorem. We assert that $\mu^{\prime} \in \Gamma^{\prime}$. Suppose that $\mu^{\prime}=a v_{1}+(1-a) \cdot v_{2}$ where $0<a<1$ and $v_{1}, v_{2} \in \Gamma$. Since $\mu^{\prime}(A)=c$, either $v_{1}(A)=v_{2}(A)=c$ or at least one of $v_{1}(A)$ and $v_{2}(A)$ is $>c$. The second alternative being impossible, we must have $v_{1}, v_{2} \in \Gamma_{1}$ and hence $\mu^{\prime}=v_{1}=v_{2}$. We thus infer that $\mu^{\prime}$ is an extreme point of $\Gamma$.

Since $\mu(A) \leqq c=\mu^{\prime}(A)$ for all $\mu$ in $\Gamma$ it is clear that $\mu(A)=0$ for all $\mu$ in $\Gamma$. The lemma is proved.

LemmA 4.6. Let $X$ be a Borel G-space and $X^{\prime}$ the G-subspace defined by an invariant set in $\mathscr{B}$. Suppose $\mathscr{E}^{\prime}$ is nonempty. Then $\mathscr{E}$ is nonempty. If there exists a decomposition map for $X$ then there exists one for $X^{\prime}$ also.

Proof. If $\mathscr{E}^{\prime}$ is nonempty and $e^{\prime}$ is an ergodic measure on $X^{\prime}$, the measure $e$ on $\mathscr{B}$ defined by $e(A)=e^{\prime}\left(A \cap X^{\prime}\right)$ for all $A$ in $\mathscr{B}$ is clearly ergodic so that $\mathscr{E}$ is nonempty. Suppose $\beta$ is a decomposition map of $X$. Let $E^{\prime}=\{e: e \in \mathscr{E}$ and $\left.e\left(X^{\prime}\right)=1\right\}$ and let $X_{1}=\left\{x: x \in X^{\prime}, \beta_{x} \in E^{\prime}\right\}$. By Lemma 4.2, $X^{\prime}-X_{1} \in \overline{\mathscr{N}}$. If we now define $\beta_{x}^{\prime}$ as the restriction of $\beta_{x}$ to $X_{1}$ whenever $x$ is in $X_{1}$ and as $e_{0}^{\prime}$ whenever $x$ is in $X^{\prime}-X_{1}, e_{0}^{\prime}$ being a fixed element of $\mathscr{E}^{\prime}$, it is easily checked that $\beta^{\prime}$ is a decomposition map of $X^{\prime}$.

THEOREM 4.2. Let $X$ be a standard Borel G-space and let $\mathscr{I} \neq \varnothing$. Then $\mathscr{E} \neq \varnothing$ and there exists an essentially unique decomposition map of $X$. Moreover, $\mathscr{E}$ is a standard Borel space.

Proof. By Theorem $3.2 X$ can be regarded as the $G$-subspace defined by an invariant Borel set of a compact metric $G$-space $Y$. Let $\mathscr{I}_{Y}$ and $\mathscr{E}_{Y}$ respectively be the spaces of invariant and ergodic measures on $Y$.

$G$ acts continuously on $Y$ and hence $\mathscr{I}_{Y}$ is a compact convex subset of the 
topological vector space of real-valued countably additive set functions on the Borel sets of $Y$. To prove that $\mathscr{E} \neq \varnothing$ it is enough to exhibit $e \in \mathscr{E}_{Y}$ with $e(X)=1$. Since $\mathscr{I} \neq \emptyset$ there are measures $v \in \mathscr{I}_{Y}$ with $v(X)>0$ and hence by Lemma 4.5 there are measures $e \in \mathscr{E}_{Y}$ with $e(X)>0$. Since $e$ is ergodic and $X$ is invariant, $e(X)=1$.

We notice also that $\mathscr{E}$ is isomorphic in a natural manner to the Borel subspace of $\mathscr{E}_{Y}$ defined by the set $E=\left\{e: e \in \mathscr{E}_{Y}, e(X)=1\right\}$. Now the set of all measures on $Y$ is a compact metric space so that $\mathscr{I}_{Y}$ is a metrizable compact convex set. By a well-known result [7] we conclude that $\mathscr{E}_{Y}$ is a $G_{\delta}$ in $\mathscr{I}_{Y}$. From this we deduce that $E$ is a Borel set in $\mathscr{I}_{Y}$ and hence that $\mathscr{E}$ is a standard Borel space.

It now remains to establish the existence of a decomposition map for $X$. In view of Lemma 4.6 we may assume that $X=Y$. In other words we shall assume, for the rest of the proof, that $X$ is itself a compact metric $G$-space. Let $G_{0}$ be a fixed countable dense subgroup of $G$. Let $C$ be the space of continuous functions on $X$ and $M$ a fixed denumerable dense subset of $C$ containing constants and such that (i) if $f_{1}, f_{2} \in M$ and $a_{1}, a_{2}$ are rationals, $a_{1} f_{1}+a_{2} f_{2} \in M$, (ii) if $f \in M$ and $g \in G_{0}$ then $L_{g} f \in M$ where $\left(L_{g} f\right)(x)=f\left(g^{-1}(x)\right)$ for all $x$. Recall that $\overline{\mathscr{N}}$ s the class of all invariant sets $A$ in $\mathscr{B}$ such that $\mu(A)=0$ for all $\mu$ in $\mathscr{I}$.

Let $U\left(f \rightarrow f^{*}\right)$ be a mapping of $\mathscr{F}$ into $\mathscr{F}$ having the properties described in Theorem 4.1. Since for each $\mu$ in $\mathscr{I}, U f=E_{\mu}(f \mid \overline{\mathscr{B}}) \mu$-almost everywhere, it follows that for $f_{1}, f_{2}$ in $M$ and rationals $a_{1}, a_{2}$,

$$
\left\{x:\left(a_{1} f_{1}+a_{2} f_{2}\right)^{*}(x) \neq a_{1} f_{1}^{*}(x)+a_{2} f_{2}^{*}(x)\right\} \in \overline{\mathscr{N}}
$$

Consequently there exists a set $N_{0} \in \overline{\mathscr{N}}$ such that

$$
\left(U\left(a_{1} f_{1}+a_{2} f_{2}\right)\right)(x)=a_{1}\left(U f_{1}\right)(x)+a_{2}\left(U f_{2}\right)(x)
$$

for all $x$ in $X-N_{0}, f_{1}, f_{2}$ in $M$, and rationals $a_{1}, a_{2}$. Since $G_{0}$ is countable we infer, from the remark following Theorem 4.1, the existence of a set $N_{1} \supseteq N_{0}$ such that $N_{1} \in \overline{\mathscr{N}}$ and $\left(U L_{g} f\right)(x)=(U f)(x)$ for all $x$ in $X-N_{1}, g$ in $G_{0}$ and $f$ in $M$. Since $\|U f\| \leqq\|f\|$ and $U 1=1$ we conclude that for each $x$ in $X-N_{1}$, the map $f \rightarrow(U f)(x)$ is the restriction to $M$ of a unique bounded linear functional $t_{x}$ on $C$ with $t_{x}(1)=1$ and $\left\|t_{x}\right\| \leqq 1 . X$ being a compact metric $G$-space, the map $(g, f) \rightarrow L_{g} f$ is continuous from $G \times C$ to $C$ and since $t_{x}\left(L_{g} f\right)=t_{x}(f)$ for all $f$ in $M$ and $g$ in $G_{0}$ we may conclude that $t_{x}\left(L_{g} f\right)=t_{x}(f)$ for all $g$ in $G$ and $f$ in $C$. By a similar argument we conclude that $t_{g(x)}(f)=t_{x}(f)$ for all $g$ in $G$ and $f$ in $C$.

Write now, for $f$ in $C$,

$$
(V f)(x)= \begin{cases}t_{x}(f) & \text { if } x \in X-N_{1} \\ 0 & \text { if } x \in N_{1} .\end{cases}
$$


For any $f$ in $M$ and $\mu$ in $\mathscr{I}$ we have $\int_{A} f d \mu=\int_{A} U f d \mu=\int_{A} V f d \mu$ for all $A \in \overline{\mathscr{B}}$. Since $f \rightarrow(V f)(x)$ is continuous in $f$ for each $x$ it follows that $\int_{A} f d \mu=\int_{A} V f d \mu$ for all $f$ in $C$ and $A \in \overline{\mathscr{B}}$, so that $V f=E_{\mu}(f \mid \overline{\mathscr{B}})$. Consequently, if $f \geqq 0$ then $(V f$ being an invariant function in any case) $\{x:(V f)(x)<0\} \in \overline{\mathscr{N}}$, so that a routine separability argument yields the existence of a set $N_{2} \supseteq N_{1}$ such that $N_{2} \in \mathcal{V} \bar{V}$ and $(V f)(x) \geqq 0$ whenever $x$ is in $X-N_{2}$ and $f$ in $C$ with $f \geqq 0$. In other words, for each $x \in X-N_{2}, f \rightarrow t_{x}(f)$ is a non-negative linear functional on $C$ with $t_{x}(1)=1$.

It now follows from the Riesz theorem that for each $x$ in $X-N_{2}$ there exists a measure $\beta_{x}$ on $X$ such that $t_{x}(f)=\int_{X} f d \beta_{x}$ for all $f$ in $C$. Since $t_{x}\left(L_{g} f\right)=t_{x}(f)$ and $t_{g(x)}(f)=t_{x}(f)$, we deduce that each $\beta_{x}$ is in $\mathscr{I}$ and $\beta_{g(x)}=\beta_{x}$ for all $x$ in $X-N_{2}$ and $g$ in $G$.

For each $f$ in $M, t_{x}(f)=(U f)(x)$ so that the function $x \rightarrow \int_{X} f(y) d \beta_{x}(y)$ is measurable for all $f$ in $M$. Since $\mathscr{F}$ is the smallest class of bounded functions containing $M$ and closed under bounded pointwise convergence, it follows that $x \rightarrow \int_{X} f(y) d \beta_{x}(y)$ is measurable for each $f$ in $\mathscr{F}$. In particular, the functions $x \rightarrow \beta_{x}(A)$ are measurable for each $A$ in $\mathscr{B}$. This shows that $x \rightarrow \beta_{x}$ is a measurable map of $X-N_{2}$ into $\mathscr{I}$.

Since $\int_{X} f d \mu=\int_{X}(U f) d \mu$ for each $\mu$ in $\mathscr{I}$ we have the equation

$$
\int_{X} f d \mu=\int_{X-N_{2}}\left(\int_{X} f(y) d \beta_{x}(y)\right) d \mu(x)
$$

valid for all $f$ in $M$. By an easy argument we conclude that it is valid for all $f$ in $\mathscr{F}$. In particular, for any $\mu$ in $\mathscr{I}$ and $A$ in $\mathscr{B}$,

$$
\mu(A)=\int_{X-N_{2}} \beta_{x}(A) d \mu(x)
$$

Suppose now $e$ is an ergodic measure. Since $e$ is two-valued over $\overline{\mathscr{B}}$, $E_{e}(f \mid \overline{\mathscr{B}})=\int_{X} f d e$ e-almost everywhere for each $f$ and hence $t_{x}(f)=\int_{X} f d e$ for $e$-almost all $x$. Since $M$ is countable there exists a set $B \supseteq N_{2}$ such that $e(B)=0$ and $t_{x}(f)=\int_{X} f d e$ for all $x$ in $X-B$ and $f$ in $M$. Since $M$ is dense in $C$ this means that $\beta_{x}=e$ for all $x$ in $X-B$. If we write $\tilde{X}_{e}=\left\{x: x \in X-N_{2}, \beta_{x}=e\right\}$ then $\tilde{X}_{e} \supseteq X-B$ so that $e\left(\tilde{X}_{e}\right)=1$.

Write now $N=\left\{x: x \in X-N_{2}, \beta_{x} \in \mathscr{I}-\mathscr{E}\right\}$. Since $\mathscr{E}$ is a Borel set in $\mathscr{I}, N$ is in $\mathscr{B}$ and is actually invariant. Since $\widetilde{X}_{e} \cap N=\varnothing$ for each $e$ in $\mathscr{E}$, it follows from the result established in the preceding paragraph that $e(N)=0$ for all $e$. Lemma 4.5 now allows us to infer that $N \in \overline{\mathscr{N}}$.

If we now define $\beta_{x}=e_{0}$ for some fixed $e_{0}$ in $\mathscr{E}$ for all $x$ in $N$, it is clear (cf. (*)) that $\beta\left(x \rightarrow \beta_{x}\right)$ is a decomposition map of $X$. Since essential uniqueness has already been established in Lemma 4.4, the proof of the theorem is complete.

The decomposition theorem leads at once to the following result.

THEOREM 4.3. Any standard Borel G-space is isomorphic to a Borel G-space 
$Y$ having the following properties: (i) the underlying Borel space is the Borel subspace of the Euclidean plane defined by a Borel set $Y \subseteq$ the unit square such that $D=\{u:(u, v) \in Y$ for some $v\}$ is a Borel subset of the unit interval, (ii) for each $u$ in $D$, the vertical section $Y_{u}$ determined by $u$ is invariant under $G$ and the G-subspace defined by $Y_{u}$ is uniquely ergodic with unique invariant measure $e_{u}$, (iii) an invariant measure $\mu$ on $Y$ is ergodic if and only if $\mu\left(Y_{u}\right)=1$ for some $u$ and $\mu=e_{u}$ over the class of Borel subsets of $Y_{u}$.

Proof. Let $\beta$ be a decomposition map of $X$. Since $X$ and $\mathscr{E}$ are both standard there are isomorphisms $\zeta$ and $\eta$ of $X$ and $\mathscr{E}$ respectively onto Borel subsets $E$ and $D$ of the unit interval. Let $\xi$ be the map $x \rightarrow\left(\zeta(x), \eta\left(\beta_{x}\right)\right)$. $\xi$ is a measurable map $X$ onto a subset $Y$ of the unit square. Since $X$ is standard and $\xi$ is one-to-one, $Y$ is a Borel set and $\xi$ is an isomorphism between $X$ and $Y$. For any $u \in D$ define $e_{u}$ as the measure induced by $\eta^{-1}(u) \in \mathscr{E}$ via the map $\zeta$, i.e., $e_{u}(A)=\eta^{-1}(u)\left(\zeta^{-1}(A)\right)$ for all Borel subsets $A$ of $Y_{u}$. Finally define $g(y)=\xi g \xi^{-1}(y)$ for all $g$ and $y$. Verification of the properties of $Y, D, Y_{u}$ and $e_{u}$ is omitted.

REMARK. A theorem similar to the above can be proved for analytic Borel $G$-spaces. We do not prove it however since the extra technical work involved in handling the analytic nonstandard case seems hardly worth the trouble. In $\S 6$ we shall give examples to illustrate the fact that Theorem 4.2 may fail to be true if the Borel structure of $X$ is not well behaved enough.

The decomposition of a standard Borel $G$-space into uniquely ergodic components has several consequences one of which is the fact that every invariant measure can be represented as an integral of ergodic measures. If the $G$-space is a compact metric one then the representation is an immediate consequence of well-known results of Choquet-Bishop-De Leeuw [3; 7]. However, as we shall see presently the powerful decomposition theory developed here is also able to take care of this situation. It is necessary to point out that the representation of invariant measures as integrals of ergodic measures is in general only dependent on the properties of the set of all invariant measures and not so much on the action of the group. In $\S 6$ we shall sketch an example of A. N. Kolmogorov which shows that there are many situations in which integral representations of invariant measures can be established but in which the decomposition theorem may fail to be valid.

We also point out that Lemma 4.5 is an immediate consequence of the ChoquetBishop-De Leeuw theorems. We have given the present proof of this lemma in view of its simplicity.

We now obtain the representation of invariant measures on standard Borel $G$ spaces. In $\S 5$ (Theorems 5.3 and 5.4) we shall obtain more general results which place Theorem 4.4 in its proper setting.

Theorem 4.4. Let $X$ be a standard Borel G-space. Then $\mathscr{E}$ is a standard Borel space and there is a one-to-one correspondence $\mu \rightarrow \sigma_{\mu}$ between the set of all invariant measures on $X$ and the set of all measures on $\mathscr{E}$ such that 


$$
\mu(A)=\int_{\delta} e(A) d \sigma_{\mu}(e)
$$

for each invariant measure $\mu$ on $X$ and all $A$ in $\mathscr{B}$.

Proof. We may assume $\mathscr{I}$ to be nonempty. Then $\mathscr{E} \neq \varnothing$ and by Theorem 4.2 $\mathscr{E}$ is a standard space. Let $\beta$ be a decomposition map of $X$ and for any invariant measure $\mu$ on $X$, define $\sigma_{\mu}(E)=\mu\left(\beta^{-1}(E)\right)$ for all $E \in \mathscr{A} . \sigma_{\mu}$ is a measure on $\mathscr{E}$ and for any $A$ in $\mathscr{B}$,

$$
\begin{aligned}
\mu(A) & =\int_{X} \beta_{x}(A) d \mu(x) \\
& =\int_{\delta} e(A) d \sigma_{\mu}(e) .
\end{aligned}
$$

Finally, if $\sigma^{\prime}$ is any measure on $\mathscr{A}$ such that $\mu(A)=\int_{\mathscr{\delta}} e(A) d \sigma^{\prime}(e)$ for all $A$ in $\mathscr{B}$, we have, for any $E$ in $\mathscr{A}, \mu\left(\beta^{-1}(E)\right)=\int_{\mathscr{\delta}} e\left(\beta^{-1}(E)\right) d \sigma^{\prime}(e)=\int_{E} d \sigma^{\prime}(e)=\sigma^{\prime}(E)$ so that $\sigma=\sigma^{\prime}$. This proves the theorem.

5. The set of invariant measures in the general case. In this section we shall prove a few relatively simple theorems on the set of invariant measures in the case of a general Borel $G$-space. All the three theorems are based on Theorem 4.1 which is the substitute, in our general context, for the Birkhoff ergodic theorem.

Suppose $\mu$ is any invariant measure. If we observe the equation $\mu(g[A])=\mu(A)$ expressing the invariance of $\mu$,it is intuitively clear that $\mu$ can have more or less arbitrary values on the class of invariant sets. The first theorem of this section makes this idea more precise and shows moreover that an invariant measure is already determined as soon as it is given on the class of invariant sets.

THEOREM 5.1. Let $X$ be a Borel G-space with underlying Borel structure $\mathscr{B}$. Let $\overline{\mathscr{B}}$ be the class of invariant sets in $\mathscr{B}$. Then if two invariant measures are equal over $\overline{\mathscr{B}}$ they are equal over $\mathscr{B}$. Moreover, if $v$ is any measure over $\overline{\mathscr{B}}$ such that $v(N)=0$ for all $N \in \bar{N}$, there exists an invariant measure (necessarily unique) $\mu$ on $\mathscr{B}$ such that $\mu(A)=v(A)$ for all $A \in \overline{\mathscr{B}}$.

Proof. Let $U$ be a mapping of $\mathscr{F}$ into $\mathscr{F}$ having the properties described in Theorem 4.1. We shall prove first the uniqueness.

Suppose $\mu_{1}$ and $\mu_{2}$ are two invariant measures which coincide on $\overline{\mathscr{B}}$. Then for any bounded $\overline{\mathscr{B}}$-measurable function $u, \int_{X} u d \mu_{1}=\int_{X} u d \mu_{2}$. If $A$ in $\mathscr{B}$ is arbitrary, the function $U \psi_{A}$ is $\overline{\mathscr{B}}$-measurable and hence we have

$$
\mu_{1}(A)=\int_{X}\left(U \psi_{A}\right) d \mu_{1}=\int_{X}\left(U \psi_{A}\right) d \mu_{2}=\mu_{2}(A) .
$$

We now prove that if $v$ is any measure on $\overline{\mathscr{B}}$ such that $v(N)=0$ for all $N \in \overline{\mathscr{N}}$, there exists an invariant measure $\mu$ on $\mathscr{B}$ such that $\mu(A)=v(A)$ for all $A$ in $\overline{\mathscr{B}}$. 
Suppose $v$ is given. For any invariant measure $\mu^{\prime}$ and any $f$ in $\mathscr{F}, U f=E_{\mu^{\prime}}(f \mid \overrightarrow{\mathscr{B}})$, and hence from the basic properties of $E_{\mu^{\prime}}(\cdot \mid \overline{\mathscr{B}})[9 ; 16]$ we conclude that the following relations hold $\mu^{\prime}$-almost everywhere:

$$
\begin{aligned}
U\left(f_{1}+f_{2}\right) & =U f_{1}+U f_{2} \\
U L_{g} f & =U f \\
U f_{n} & \searrow 0 \quad \text { if } f_{n} \searrow 0 \\
U \bar{f} & =\bar{f} ;
\end{aligned}
$$

here $f, f_{1}, f_{2} \cdots$ are in $\mathscr{F},\left(L_{g} f\right)(x)=f\left(g^{-1}(x)\right)$ and $\bar{f}$ is $\overline{\mathscr{B}}$-measurable. For fixed $f, f_{1}, \cdots$ the sets of points of $X$ where the relations in $\left({ }^{*}\right)$ do not hold are independent of $\mu^{\prime}$ so that these sets lie in $\mathscr{N}$. Moreover the functions involved in the above equations are all $\overline{\mathscr{B}}$-measurable so that these sets are also in $\overline{\mathscr{B}}$. Consequently the sets in question belong to $\overline{\mathscr{N}}$ and hence are of $v$-measure zero. If we now define for all $A$ in $\mathscr{B}$,

$$
\mu(A)=\int_{X}\left(U \psi_{A}\right) d v
$$

then, the fact that the relations $\left({ }^{*}\right)$ hold $v$-almost everywhere leads to the conclusion that $\mu$ is an invariant measure which coincides with $v$ on $\overline{\mathscr{B}}$. This proves the theorem.

REMARK. The part of the above theorem asserting that an invariant measure is determined by its values on $\overline{\mathscr{B}}$ was pointed out by J. Blum and D. L. Hanson [5] in the special case when $G$ is the discrete additive group of all integers.

The class $\overline{\mathscr{N}}$ is a $\sigma$-ideal in $\overline{\mathscr{B}}$. We may thus form the quotient $\sigma$-algebra $R=\overline{\mathscr{B}} / \overline{\mathscr{N}}$. Clearly there is a natural one-to-one correspondence between the measures on $R$ and the measures on $\overline{\mathscr{B}}$ which vanish over $\overline{\mathscr{N}}$. Given two $\sigma$ algebras $R_{1}$ and $R_{2}$ and two convex sets of measures $\Gamma_{1}$ and $\Gamma_{2}$ over $R_{1}$ and $R_{2}$ respectively, we shall say that $\Gamma_{1}$ and $\Gamma_{2}$ are isomorphic if there exists a one-toone correspondence $\tau: \mu \rightarrow \tau_{\mu}$ between $\Gamma_{1}$ and $\Gamma_{2}$ such that if $\mu_{n} \rightarrow \tau_{\mu_{n}}=v_{n}$ for $n=1,2, \cdots$ and $\mu_{0}=c_{1} \cdot \mu_{1}+c_{2} \cdot \mu_{2}+\cdots$ where $c_{1}, c_{2}, \cdots$ are constants $\geqq 0$ with $c_{1}+c_{2}+\cdots=1$ and $\mu_{0} \in \Gamma_{1}$, then $\tau_{\mu_{0}}=v_{0}$ where $v_{0}=c_{1} \cdot v_{1}+c_{2} \cdot v_{2}+\cdots$.

We then have the following easy consequence of Theorem 5.1, which is a generalization of Theorem 4.4 to the case of a general Borel $G$-space.

THEOREM 5.2. Let $X$ be a Borel G-space and $\mathscr{I}$ the set of all invariant measures. Then there exists a $\sigma$-algebra $R$ such that $\mathscr{I}$ is isomorphic to the set of all measures on $R$.

We end this section with a theorem which gives, in the general case, a necessary and sufficient condition that every invariant measure can be represented as an integral of ergodic measures. Every analytic Borel space will be shown to satisfy this condition so that this theorem includes Theorem 4.4. We shall say that a 
$G$-space $X$ has sufficiently many ergodic measures if for any $A$ in $\overline{\mathscr{B}}, \mu(A)=0$ for all invariant measures $\mu$ if and only if $e(A)=0$ for all ergodic measures $e$.

THEOREM 5.3. If $X$ is an analytic Borel G-space, then $X$ has sufficiently many ergodic measures. Moreover, in this case, the space $\mathscr{E}$ of ergodic measures is an analytic Borel space.

Proof. By Theorem 3.2 we may assume that $G$-space $X$ is isomorphic to a $G$-subspace of a compact metric $G$-space $X^{\prime}$, defined by an analytic invariant set $\tilde{X}$. We may assume that $\mathscr{I} \neq \varnothing$. Let $\xi$ be an isomorphism of $X$ with $\tilde{X}$. We denote by $G_{0}$ a countable dense subgroup of $G$. Since $G$ acts on $X^{\prime}$ continuously, the set $\mathscr{I}^{\prime}$ of invariant measures coincides with the set of measures invariant under the action of the elements of $G_{0}$.

Suppose $A \subseteq X$ and $e(A)=0$ for all $e \in \mathscr{E}$. Suppose that for some invariant measure $\mu, \mu(A)>0$. If $\mu^{\prime}(E)=\mu\left(\xi^{-1}(E)\right)$ for all Borel sets $E$, then $\mu^{\prime}$ is an invariant measure and $\mu^{\prime *}(\xi[A])=\mu(A)>0$. Since $\xi[A]$ is an analytic subset of $X$ it is $\mu^{\prime *}$-measurable so that there exists a Borel subset $B \subseteq \xi[A]$ such that $\mu^{\prime}(B)=\mu^{\prime *}(\xi[A])=\mu(A)>0$. Since we can replace $B$ by $\bigcup_{g \in G_{0}} g[B]$, we may (and do) assume that $B$ is $G_{0}$-invariant. By Lemma 4.5 there exists an ergodic measure $e_{0}^{\prime}$ of $X^{\prime}$ such that $e_{0}^{\prime}(B)>0$. Since $B$ is $G_{0}$-invariant it follows that $e_{0}^{\prime}(B)=1$. In particular, $e_{0}^{\prime *}(\tilde{X})=1$ so that there exists a measure $e_{0}$ on $X$ such that $e_{0}^{\prime}(E)=e_{0}\left(\xi^{-1}(E)\right)$ for all Borel sets $E$. $e_{0}$ is clearly ergodic and $e_{0}(A) \geqq e_{0}\left(\xi^{-1}(B)\right)=e_{0}^{\prime}(B)>0$. This contradiction proves that $X$ must have sufficiently many ergodic measures.

It is easily seen that the Borel space $\mathscr{M}$ of all measures on $X$ is isomorphic to the subset $\tilde{\mathscr{M}}$ of the space $\mathscr{M}^{\prime}$ of all measures on $X^{\prime}$ consisting of all measures $v^{\prime}$ with $v^{\prime *}(\tilde{X})=1$. By Theorem 2.1, $\tilde{\mathscr{M}}$ is an analytic subset of $\mathscr{M}^{\prime}$. Moreover $\mathscr{E}$ is isomorphic to $\mathscr{E}^{\prime} \cap \tilde{\mathscr{M}}$. Since $\mathscr{E}^{\prime}$ is a Borel set in $\mathscr{M}^{\prime}$ it follows that $\mathscr{E}^{\prime} \cap \tilde{\mathscr{M}}$ is an analytic subset of $\mathscr{M}^{\prime}$. This shows that $\mathscr{E}$ is an analytic Borel space and completes the proof of the theorem.

We can now formulate our final theorem in this section. Let $X$ be a Borel $G$-space and for each $M \in \overline{\mathscr{B}}$ let

$$
\tau(M)=\{e: e \in \mathscr{E}, e(M)=1\} .
$$

$\tau(M) \subseteq \mathscr{E}$ and is a set in the Borel structure $\mathscr{A}$ for $\mathscr{E}$. An invariant measure $\mu$ will be said to be representable if there exists a measure $\sigma$ on $(\mathscr{E}, \mathscr{A})$ such that

for all $A \in \mathscr{B}$.

$$
\mu(A)=\int_{\mathscr{E}} e(A) d \sigma(e)
$$

THEOREM 5.4. A necessary and sufficient condition that every invariant measure be representable is that $X$ have sufficiently many ergodic measures. 
If this condition is satisfied, then $\tau(M \rightarrow \tau(M))$ is a $\sigma$-homomorphism of the Boolean $\sigma$-algebra $\overline{\mathscr{B}}$ onto the Boolean $\sigma$-algebra $\mathscr{A}$ having as kernel the $\sigma$ ideal $\overline{\mathscr{N}}$. Moreover, if $\mu$ is any invariant measure and we define $\sigma(\tau(M))=\mu(M)$ for any $M \in \overline{\mathscr{B}}, \sigma$ is a well-defined measure on $(\mathscr{E}, \mathscr{A})$ and is the unique measure such that

$$
\mu(A)=\int_{\mathscr{E}} e(A) d \sigma(e)
$$

for all $A$ in $\mathscr{B}$.

Proof. It is trivial that if every invariant measure $\mu$ is representable, then $X$ has sufficiently many ergodic measures. Suppose now $X$ has sufficiently many ergodic measures. It is then easily shown that $\tau$ is a $\sigma$-homomorphism of $\overline{\mathscr{B}}$ into $\mathscr{A}$ with kernel $\overline{\mathscr{N}}$. We claim that $\tau$ maps $\overline{\mathscr{B}}$ onto $\mathscr{A}$. To prove this, it is enough to prove that if $A$ is in $\mathscr{B}$ and $0 \leqq c \leqq 1,\{e: e(A) \leqq c\}$ lies in the range of $\tau$. Let $f^{*}=U \psi_{A}$ where $U$ is a mapping of $\mathscr{F}$ into $\mathscr{F}$ having the properties described in Theorem 4.1 and define $M=\left\{x: f^{*}(x) \leqq c\right\}$. It is easily checked that $\tau(M)=\{e: e(A) \leqq c\}$. In fact, if $e(M)=1$, then $e(A)=\int_{X} \psi_{A} d e=\int_{X} f^{*} d e=\int_{M} f^{*} d e \leqq c$; while $e(M)<1$ implies, since $M$ is invariant, that $e(M)=0$ and hence

$$
e(A)=\int_{X} \psi_{A} d e=\int_{X} f^{*} d e=\int_{X-M} f^{*} d e>c .
$$

Finally, since the kernel of $\tau$ is $\overline{\mathscr{N}}$, given any invariant measure $\mu$, the function $\sigma$ is well-defined on setting $\sigma(\tau(M))=\mu(M)$ for all $M$ in $\overline{\mathscr{B}} . \sigma$ is obviously a measure on $\mathscr{A}$. Let $\mu^{\prime}$ be the measure on $\mathscr{B}$ defined by $\mu^{\prime}(A)=\int_{\mathscr{E}} e(A) d \sigma(e)$ for all $A$ in $\mathscr{B}$. $\mu^{\prime}$ is invariant and coincides with $\mu$ on $\overline{\mathscr{B}}$. Consequently, it can be concluded from Theorem 5.1 that $\mu^{\prime}=\mu$. If $\sigma^{\prime}$ is any measure on $\mathscr{A}$ such that $\mu(A)=\int_{\delta} e(A) d \sigma^{\prime}(e)$ for all $A$, then for any $M$ in $\overline{\mathscr{B}}$,

$$
\mu(M)=\int_{\mathscr{E}-\tau(M)} e(M) d \sigma^{\prime}(e)+\int_{\tau(M)} e(M) d \sigma^{\prime}(e)=\sigma^{\prime}(\tau(M))=\sigma(\tau(M)),
$$

allowing us to infer that $\sigma=\sigma^{\prime}$. This proves the theorem.

REMARK. In $\S 6$ we shall present examples of separable Borel $G$-spaces which do not have sufficiently many ergodic measures. On the other hand Lemma 4.5 shows that any compact Hausdorff $G$-space has sufficiently many ergodic measures. It seems difficult to formulate conditions, analogous to that of Theorem 5.3, in the general case which would ensure the existence of sufficiently many ergodic measures.

6. Examples. In this section we shall present a number of examples illustrating various aspects of the foregoing theory.

1. Given any countable group $G$, there exists an uncountable compact metric $G$-space $X$ on which there exist nonatomic invariant measures. 
Let $Y$ be any uncountable compact metric space and let $X$ be the space of all functions from $G$ to $Y$. Equipped with the product topology, $X$ becomes a compact metric space. For any $g$ in $G$ and $x$ in $X$ we define $g(x)$ to be the element of $X$ given by $g(x)(h)=x(h g)$ for all $h \in G$. The map $(g, x) \rightarrow g(x)$ is continuous in $g$ and $x$ ( $G$ has the discrete topology) so that $X$ is a $G$-space. Given any measure $\mu$ on $Y$ we define the measure $\mu^{G}$ on ${ }_{i}^{\prime} X$ to be the iunique measure of Borel subsets of $X$ such that for all distinct $g_{1}, g_{2}, \cdots, g_{k}$ in $G$ and Borel sets $A_{1}, \cdots, A_{k} \subseteq Y$,

$$
\mu_{G}(B)=\prod_{i=1}^{k} \mu\left(A_{i}\right)
$$

where $B=\left\{x: x \in X, x\left(g_{i}\right) \in A_{i}\right.$ for all $\left.i=1,2, \cdots, k\right\} . \mu^{G}$ is clearly invariant. If $\mu$ is nontrivial on $Y, \mu^{G}$ is even nonatomic.

2. Examples of standard Borel $G$-spaces supporting nonatomic ergodic measures also arise in the theory of stationary stochastic processes. Let $G$ be the additive group of vectors in $n$-dimensional space and $X$ the space of continuous functions on $G$. Equipped with the topology of uniform convergence on compact sets $X$ becomes a complete separable metric space. For any $\dot{g}$ in $G$ and $x$ in $X$ we define $g(x)$ to be the function $h \rightarrow x\left(g^{-1} h\right)$ on $G$. Under the action $(g, x) \rightarrow g(x), X$ becomes a Borel $G$-space. It can be shown that there are nonatomic ergodic measures on $X$ (cf. [9] for the elements of the theory of stationary stochastic processes). When $G$ is the additive group of real numbers the invariant measures on $X$ correspond to the so-called stationary stochastic processes with continuous sample functions.

Suppose now that $G$ is the noncompact, non-abelian group of all matrices

$$
g=\left(\begin{array}{cc}
a & b \\
0 & 1 / a
\end{array}\right)
$$

where $a>0$ and $-\infty<b<\infty$. Since the map $\psi: g \rightarrow \log a$ is a continuous homomorphism of $G$ onto the additive group $R^{1}$ of real numbers, the map $f \rightarrow f \circ \psi$ is an isomorphism of the space $X\left(R^{1}\right)$ of all continuous functions on $R^{1}$ into the space $X(G)$ of all continuous functions on $G$ such that it exchanges the natural action of $R^{1}$ on $X\left(R^{1}\right)$ with the natural action of $G$ on $X(G)$. Since there are ergodic nonatomic measures on $X\left(R^{1}\right)$, it follows that there are nonatomic measures on $X(G)$ which are ergodic under the natural action of $G$ on $X(G)$. Note that the natural action of $G$ on $X(G)$ converts $X(G)$ into a Borel $G$-space.

3. Given any infinite countable group $G$ there exists a uniquely ergodic standard $G$-space $X$ on which $G$ acts freely, i.e., for any $x \in X, g(x)=x$ implies that $g$ is the identity of $G$.

Let us take any uncountable compact metric space $Y$ and let us consider the compact metric $G$-space $X^{\prime}$ of all functions from $G$ to $Y$ constructed in 1 . above. Write $X_{0}=\left\{x: x \in X^{\prime}, x\right.$ is one-to-one on $\left.G\right\} . X_{0}$ is :an invariant Borel set and 
$\mu^{G}\left(X_{0}\right)=1$ for any nonatomic measure $\mu$ on $Y$. G acts freely on $X_{0}$. Using Theorem 4.3 we can find an invariant Borel set $X^{\prime} \subseteq X_{0}$ such that the $G$-subspace defined by $X^{\prime}$ is uniquely ergodic. Since $G$ is infinite and acts freely on $X^{\prime}$, the unique invariant measure on $X^{\prime}$ is necessarily nonatomic.

4. Given any infinite countable group $G$ there exists a separable $G$-space $X$ on which there exist continuum many invariant measures but no ergodic measures.

To construct such an $X$ we start off with the $G$-space $X^{\prime}$ constructed in 3. above with the unique invariant measure $\mu$. Since $X^{\prime}$ is standard and $\mu$ is nonatomic we may (and do) assume that $X^{\prime}$ is the unit interval and that $\mu$ is Lebesgue measure on $X^{\prime}$. Let $W=X^{\prime} \times X^{\prime}$ and for $g$ in $G$ and $\left(x, x^{\prime}\right)$ in $W$, let

$$
g\left(x, x^{\prime}\right)=\left(x, g\left(x^{\prime}\right)\right) .
$$

Equipped with the usual Borel structure, $W$ thus becomes a Borel $G$-space. The required $G$-space $X$ will be the $G$-subspace of $W$ defined by a suitably chosen invariant subset of $W$. Let $\pi$ be a Lebesgue measure on $W$. For any $E \subseteq W$ and any $x$ in $X^{\prime}$ let $E_{x}=\{y:(x, y) \in E\}$ and $E^{x}=\{y:(y, x) \in E\}$. We need a lemma.

Lemma 6.1. There exists a set $Q \subseteq W$ such that $\pi^{*}(Q)=1$ and $Q_{x}$ consists of precisely one point for each $x$ in $X^{\prime}$.

Proof. Let $K$ be the set of all sequences $a_{\sim}=\left(a_{1}, a_{2}, \cdots\right)$ where each $a_{j}$ is 0 or 1 . By the classical construction of Lebesgue, there exist sets $M_{0}$ and $M_{1} \subseteq X^{\prime}$ such that $M_{0} \cap M_{1}=\varnothing, M_{0} \cup M_{1}=X^{\prime}$ and $\mu^{*}\left(M_{0}\right)=\mu^{*}\left(M_{1}\right)=1$. Let $X$ be the cartesian product of countably many copies of $X^{\prime}$ and $\tilde{\mu}$ the product measure on $\widetilde{X}$. For any $a_{\sim}=\left(a_{1}, a_{2}, \cdots\right)$ in $K$ we write

$$
M_{a_{\sim}}=\prod_{j=1}^{\infty} M_{a_{j}},
$$

where $\prod$ denotes the cartesian product. It is easily shown that $\tilde{\mu}^{*}\left(M_{a_{\sim}}\right)=1$ for all $a_{\sim}$. The $M_{a_{\sim}}$ are mutually disjoint Borel sets in $\tilde{X}$ and $\bigcup_{a_{\sim} \in K} M_{a_{\sim}}=\tilde{X}$. Since there is an isomorphism between $\tilde{X}$ and $X$ that exchanges $\tilde{\mu}$ and $\mu$, and since there are continuum many $a_{\sim}$ in $K$, we conclude that there exists a family $\left\{M_{t}: 0 \leqq t \leqq 1\right\}$ of mutually disjoint subsets of $X^{\prime}$ such that $\bigcup_{0 \leqq t \leqq 1} M_{t}=X^{\prime}$ and $\mu^{*}\left(M_{t}\right)=1$ for all $t$. Define now

$$
Q=\left\{(x, t): x \in M_{t}\right\} .
$$

Since for any $x$ in $X^{\prime}$, there exists precisely one $t$ in $[0,1]$ such that $x \in M_{t}$, it follows that $Q_{x}$ consists of a single point for each $x$ in $X^{\prime}$. Moreover, for each $t \in X^{\prime}=[0,1], Q^{t}=M_{t}$ has $\mu$-outer measure 1 , so that we may conclude from Fubini's theorem that $\pi^{*}(Q)=1$. This proves the lemma.

We now resume the discussion of the example. Let $Q \subseteq W$ be a set as in the lemma and let 


$$
X=\bigcup_{g \in G} g[Q] .
$$

$X$ is an invariant subset of $W$ and $X_{x}$ is a countable set for each $x$ in $X^{\prime}$. The $G$-subspace defined by $X$ is separable; and since $\pi^{*}(X)=1$ there exists a measure $v$ on $X$ such that $\pi(B)=v(B \cap X)$ for all Borel sets $B \subseteq W . v$ is clearly a nonatomic invariant measure. We claim that there exists no ergodic measure on $X$. Suppose in fact that $e$ is an ergodic measure on $X$. Then the measure $e_{0}$ defined by $e_{0}(B)=e(B \cap X)$ is an ergodic measure on $W$. It now follows easily from the construction of $W$ that there exists an $x_{0} \in X^{\prime}$ such that $e_{0}\left(W_{x_{0}}\right)=1$. This means that $e\left(W_{x_{0}} \cap X\right)=e\left(X_{x_{0}}\right)=1$. This last equation is impossible since $X_{x_{0}}$ is countably infinite. Thus there exist no ergodic measures on $X$. By restricting $v$ to various invariant subsets of positive measure we obtain continuum many invariant nonatomic measures.

5. Another class of examples arises from a consideration of the so-called permutation groups. Let $N$ be any countably infinite set and $G$ a countable group acting on $N$ so that $N$ is a $G$-space. For any Borel space $(X, \mathscr{B})$ let $\left(X^{N}, \mathscr{B}^{N}\right)$ be the (product) Borel space of all functions from $N$ to $X . X^{N}$ becomes a $G$-space if we define, for $x$ in $X^{N}$ and $g$ in $G, g(x)$ to be the function $n \rightarrow x\left(g^{-1}(n)\right)$ from $N$ to $X$. For any measure $\mu$ on $\mathscr{B}$ we write $\mu^{N}$ for the unique measure on $\mathscr{B}^{N}$ such that for distinct $n_{1}, \cdots, n_{k}$ in $N$ and $A_{1}, \cdots, A_{k}$ in $\mathscr{B}$,

$$
\mu^{N}(B)=\prod_{i=1}^{k} v\left(A_{i}\right)
$$

where $B=\left\{x: x \in X^{N}, x\left(n_{i}\right) \in A_{i}\right.$ for $\left.i=1,2, \cdots, k\right\} . \mu^{N}$ is invariant under $G$. The results of $\S \S 4$ and 5 are thus applicable whenever $(X, \mathscr{B})$ is an analytic Borel space.

Suppose now $N=G=$ the additive group of all integers and $X$ the $G$-space constructed in 4. above. For any measure $\mu$ on $\mathscr{B}$ the measure $\mu^{N}$ is now known to be even ergodic (in fact $X^{N}$ is the so-called sequence space in which $G$ acts as a shift). Thus the space of ergodic measures on $X^{N}$ is nonempty. Consider now the mapping $\tau$ of $X$ into $X^{N}$ which sends any $x$ in $X$ to the function $\tau(x)$ defined by $\tau(x)(h)=h^{-1}(x)$ for all $h \in G . \tau$ is then easily seen to be an imbedding of the $G$-space $X$ into the $G$-space $X^{N}\left(=X^{G}\right)$. Moreover, from the separability of $X$, we conclude easily that $\tau[X]$ is a set in $\mathscr{B}^{N}$. The measure $v$ on $X$ constructed in 4 . gives rise, via $\tau$, to a measure $v^{\tau}$ on $X^{N}$. Clearly $v^{\tau}$ is invariant and $v^{\tau}(\tau[X])=1$. But $v^{\tau}$ is not an integral of ergodic measures because if this were the case, there will exist ergodic measures $e$ on $X^{N}$ with $e(\tau[X])=1$; this will imply the existence of ergodic measures on $X$, a contradiction. The example of 4 . above and the example of a shift-invariant measure on $X^{N}$ which is not an integral of shiftergodic measures seem to be the first such explicit examples in the literature.

6. Suppose now that $N$ is an arbitrary countably infinite set and $G$ is the group 
all one-to-one transformations $n \rightarrow g(n)$ of $N$ such that $g(n)=n$ for all but finitely many $n$. This was considered by De Finetti [8] when $(X, \mathscr{B})$ is the Borel space associated with the real line. The results of De Finetti were extended to more general situations by Hewitt-Savage (cf. [12] and the references cited there). In this case it turns out that for any measure $\mu$ on $X, \mu^{N}$ is ergodic on $X^{N}$ and that every ergodic measure on $X^{N}$ is of the form $\mu^{N}$ for some measure $\mu$ on $\mathscr{B}$. Thus, whenever $X$ is an analytic Borel space, every invariant measure on $X$ is an integral of measures of the form $\mu^{N}$. It can be shown that this is true even when $\mathscr{B}$ is only $\sigma$-isomorphic to some sub $\sigma$-algebra of the Borel structure of an analytic Borel space. Such Borel spaces $(X, \mathscr{B})$ need not be countably generated. It seems to be difficult to construct a separable Borel space $X$ such that $X^{N}$ supports invariant measures which are not integrals of measures of the form $\mu^{N}$.

7. The decomposition theory developed in $\$ 4$ may not obtain if we omit the the hypotheses concerning $G$. A counter-example due to A. N. Kolmogorov [10] is as follows. Let $X_{0}$ be the set composed of the two points 0 and 1 and let $X$ be $X_{0}^{N}$ (cf. 5. and 6. above). For $G$ we take the group of all permutations of $N$. Let $G_{0}$ be the group of all permutations which leave all but finitely many elements of $N$ fixed. Then it is easily shown that a measure on $X^{N}$ is invariant under $G$ if and only if invariant under $G_{0}$. In particular, there are continuum many ergodic measures. There are, however, only countably many orbits. In this case every invariant measure is two-valued on the class of invariant sets. Notice that the representation theorems of Choquet-Bishop-De Leeuw apply nevertheless and lead to representations of invariant measures as integrals of ergodic measures.

8. When $X$ is an arbitrary compact metric $G$-space it may happen that there are no invariant measures. Fomin [10] has proved theorems according to which when $G$ is suitably restricted (abelian, solvable, etc.), there exist invariant measures on every compact metric $G$-space.

\section{REFERENCES}

1. W. Ambrose, P. R. Halmos and S. Kakutani, The decomposition of measures. II, Duke Math. J. 9 (1942), 43-47.

2. W. Ambrose and S. Kakutani, On the structure and continuity of measurable flows, Duke Math. J. 9 (1942), 25-42.

3. E. Bishop and $\mathrm{K}$. De Leeuw, The representations of linear functionals by measures on sets of extreme points, Ann. Inst. Fourier (Grenoble) 9 (1959), 305-331.

4. D. Blackwell, On a class of probability spaces, Proc. 3rd Berkeley Sympos. Math. Statist. and Prob., Vol. 2, pp. 1-6, Univ. of California Press, Berkeley, Calif., 1956.

5. J. R. Blum and D. L. Hanson, On invariant probability measures. I, Pacific J. Math. 10 (1960), 1125-1130.

6. D. L. Burkholder and Y. S. Chow, Iterates of conditional expectation operators, Proc. Amer. Math. Soc. 12 (1961), 490-495.

7. G. Choquet, Existence et unicité des représentations intégrales, Séminaire Bourbaki, 1956-1957, pp. 139-01 to 139-15, Secrétariat Mathématique, Paris, 1959. 
8. B. De Finetti, La prévision, ses lois logiques, ses sources subjectives, Ann. Inst. H. Poincaré 7 (1937), 1-68.

9. J. L. Doob, Stochastic processes, Wiley, New York, 1953.

10. S. V. Fomin, On measures invariant under certain groups of transformations, Izv. Akad. Nauk SSSR 14 (1950), 261-274. (Russian)

11. P. R. Halmos, Measure theory, Van Nostrand, New York, 1950.

12. E. Hewitt and L. J. Savage, Symmetric measures on cartesian products, Trans. Amer. Math. Soc. 80 (1955), 470-501.

13. $\mathrm{N}$. Krylov and $\mathrm{N}$. Bogoljubov, La théorie générale de la mesure dans son application à l'étude des systèmes dynamiques de la mécanique non linéaire, Ann. of Math. (2) 38 (1937), 65-113.

14. C. Kuratowski, Topologie. I, Espaces Métrisables, Espaces Complets, 2nd ed., Vol. 20, Monogr. Mat., Warsaw, 1948.

15. L. H. Loomis, An introduction to abstract harmonic analysis, Van Nostrand, New York, 1953.

16. M. Loève, Probability theory, Van Nostrand, New York, 1955.

17. G. W. Mackey, Borel structures in groups and their duals, Trans. Amer. Math. Soc. 85 (1957), 134-165.

18. J. von Neumann, Zur Operatorenmethode in der klassischen Mechanik, Ann. of Math. (2) 33 (1932), 587-642.

19. J. C. Oxtoby, Ergodic sets, Bull. Amer. Math. Soc. 58 (1952), 116-136.

20. Ju. V. Prohorov, Convergence of random processes and limit theorems in probability theory, Teor. Verojatnost. i Primenen 1 (1956), 177-238. (Russian)

21. V. S. Varadarajan, On the weak convergence of measures on separable metric spaces, Sankhyā 19 (1958), 15-22.

22. - Measures on topological spaces, Mat. Sb. (N. S.) 55 (97) (1961), 35-100. (Russian)

23. - On a problem in ergodic theory, Bull. Amer. Math. Soc. 68 (1962), 354-350.

\author{
NEW YORK UNIVERSITY, \\ NEW YoRK, NEW YoRK \\ Indian Statistical Institute, \\ Calcutta, India
}

